\title{
BOUNDARY SENTINELS IN CYLINDRICAL DOMAINS *
}

\author{
J. SAINT JEAN PAULIN and M. VANNINATHAN
}

\begin{abstract}
We study a model describing vibrations of a cylindrical domain with thickness $e>0$. A characteristic of this model is that it contains "pollution terms" in the boundary data and "missing terms" in the initial data. The "method of sentinels" of J.L. Lions [7] is followed to construct a sentinel using the observed vibrations on the boundary. Such a sentinel, by construction, provides information on pollution terms independent of missing terms. This requires resolution of initial-boundary value problems with nonzero boundary data of mixed type and an exact controllability problem. Further, we characterize so called "stealthy pollution terms" present in the model.
\end{abstract}

\section{Introduction}

The subject matter of this work is vibrations of three-dimensional domains of cylindrical shape $\left.\Omega^{e}=\omega \times\right]-e / 2, e / 2[, \omega$ being a planar domain. Here $e>0$ denotes the thickness of the domain in the $x_{3^{-}}$ direction. The vibrations of $\Omega^{e}$ are modelized by the wave equation. The boundary data are sums of principal terms and perturbing terms known as "pollution terms". Roughly speaking, these "pollution terms" are unknown source terms creating the vibrations. Likewise, initial data are of a similar nature and the terms of perturbation which occur here are called "missing terms". These terms of perturbation are assumed to be small. The above system provides an example of what is known as incomplete system in the literature.

\footnotetext{
${ }^{*}$ This work forms part of the Project 1001-1 sponsored by IFCPAR, New Delhi.
} 
One of the objectives in the study of such systems is to obtain information on the pollution terms which are naturally required to be "independent" of missing terms. We are thus dealing with a type of inverse problem. The idea, as in many other cases, is to observe the solution (here vibrations of $\Omega^{e}$ ) in a suitable portion of $\overline{\Omega^{e}}$ during a certain period of time. Using suitable averages of these observed data, one constructs functionals which, a priori, depend on pollution terms as well as on missing terms. It is natural to require that the first order variations of the functional w.r.t the missing terms are zero. Conditions thus obtained are referred to as "insensitivity conditions" and functionals satisfying them are called "sentinels". Thus sentinels are independent of missing terms to the first order. Once constructed, they provide useful information on the pollution terms. Insensitivity conditions lead to an exact controllability problem which we know how to treat.

Above is a short description of the method of sentinels introduced and developed in the book of Lions [7] wherein the reader can also find further the significance of pollution terms and missing terms and distinction between them. Many models occurring in practice do contain such terms and hence the study of sentinels is very important in applications. As far as our knowledge goes, there are not many articles devoted to sentinels except the work [7]. We mention also that H.U.M. (Hilbert Uniqueness Method) introduced in the books of Lions [6] can be employed fruitfully to analyze the exact controllability problem arising in the construction of the sentinel.

In our work, there are essentially three parts. The first part $(\S 3-\S 7)$ deals with initial-boundary value problems with non-zero boundary data. Our situation is slightly complicated because of the mixed nature of the boundary conditions: Dirichlet condition on the lateral portion $\Gamma_{0}^{e}$ of the boundary $\partial \Omega^{e}$ and Neumann condition on the top-bottom surfaces $\Gamma_{ \pm}^{e}$ of $\partial \Omega^{e}$. Starting from homogeneous problem, we proceed systematically and present a method of solving non-homogeneous problem and obtaining estimates independent of $e$. For the treatment of non-mixed problems, we refer the reader to [3], [4] and the references found therein.

The second part ( $\S 8)$ constructs a sentinel based on the observation of the vibrations on $\Gamma_{0}^{e}, \Gamma_{ \pm}^{e}$. The resulting exact controllability problem has already been solved by the authors [8]. Here we recall the results 
from [8] with some significant improvements.

The last part ( $\S 9)$ deals with yet another aspect of the problem: there are degenerate situations where sentinels provide no information on the pollution terms up to first order. If this happens, such pollution terms are called stealthy $([7])$. In $\S 9$, we characterize stealthy pollution terms in our problem. They appear as boundary values in some overdetermined systems. Whether they are nontrivial or not depends on the domain $\omega$.

In this work, we derive estimates (uniform w.r.t $e$ ) on various problems connected with sentinel. Their asymptotic behaviour as $e \rightarrow 0$ is the object of a forthcoming paper.

\section{Notations and problem to be studied}

We are interested in the study of vibrations of the thin cylindrical domain $\Omega^{e}$. To carry out the analysis, following the usual practice, we make the transformation $z_{\alpha}=x_{\alpha}, \alpha=1,2$ and $z_{3}=e^{-1} x_{3}$ which takes the domain $\Omega^{e}$ to a fixed domain $\left.\Omega=\omega \times\right]-1 / 2,1 / 2[$ of thickness unity. Here $\omega$ is a bounded domain in $\mathbb{R}^{2}$ with smooth boundary $\gamma$. We set

$$
\left.\Gamma_{0}=\gamma \times\right]-1 / 2,1 / 2\left[, \quad \Gamma_{ \pm}=\omega \times\{ \pm 1 / 2\}, \quad \Gamma=\Gamma_{0} \cup \Gamma_{+} \cup \Gamma_{-} .\right.
$$

We see that the boundary $\Gamma$ of $\Omega$ is partitioned into the lateral part $\Gamma_{0}$, the upper part $\Gamma_{+}$and the lower part $\Gamma_{-}$. Let $\nu=\nu(z)$ denote the outward unit normal erected at the boundary point $z \in \Gamma$.

We study the following initial-boundary value problem where the state variable $y^{e}$ represents the amplitude of vibrations of the domain $\Omega$ during a time interval $0 \leq t \leq T$ :

$$
\left\{\begin{array}{l}
\square_{e} y^{e}=0 \text { in } Q \\
y^{e}=h_{0}^{e}+\lambda_{0} \widehat{h}_{0}^{e} \text { on } \Sigma_{0}, e^{-1}\left(\partial y y^{e} / \partial \nu\right)=h_{ \pm}^{e}+\lambda_{ \pm} \widehat{h}_{ \pm}^{e} \text { on } \Sigma_{ \pm} \\
y^{e}(0)=y_{0}^{e}+\tau_{0} \widehat{y}_{0}^{e} \text { in } \Omega,\left(y^{e}\right)^{\prime}(0)=y_{1}^{e}+\tau_{1} \widehat{y}_{1}^{e} \text { in } \Omega
\end{array}\right.
$$

Here we used the following usual notations:

$$
\begin{gathered}
Q=\Omega \times] 0, T[, \quad \Sigma=\Gamma \times] 0, T[ \\
\left.\Sigma_{0}=\Gamma_{0} \times\right] 0, T\left[, \quad \Sigma_{ \pm}=\Gamma_{ \pm} \times\right] 0, T[. \\
\square_{e}=\left(\partial^{2} / \partial t^{2}\right)-\Delta_{e}, \text { where } \Delta_{e}=\left(\partial^{2} / \partial z_{\alpha} \partial z_{\alpha}\right)+e^{-2}\left(\partial^{2} / \partial z_{3}^{2}\right) .
\end{gathered}
$$


Here and in the sequel, we follow the usual summation convention with respect to the repeated indices. Greek indices take values in $\{1,2\}$.

In the above system, $\tau_{0} \widehat{y}_{0}^{e}$ and $\tau_{1} \widehat{y}_{1}^{e}$ represent the missing terms in the initial data whereas $\lambda_{0} \widehat{h}_{0}^{e}$ and $\lambda_{ \pm} \widehat{h}_{ \pm}^{e}$ are the so-called pollution terms appearing in boundary conditions. The parameters $\tau=\left(\tau_{0}, \tau_{1}\right)$ and $\lambda=\left(\lambda_{0}, \lambda_{ \pm}\right)$are supposed to be small.

The question we are concerned with is the following: can we obtain some information on pollution terms which is insensitive to the missing data, at least up to first order in $\tau_{0}, \tau_{1}$ ? For this purpose, we observe $y^{e}=y^{e}(\lambda, \tau)$ on $\Sigma_{ \pm}$and its normal trace on $\Sigma_{0}$. We then construct the functional

$$
\int_{\Sigma_{0}} \xi_{0}^{e} \frac{\partial y^{e}}{\partial \nu} d \sigma d t-\int_{\Sigma_{+}} \xi_{+}^{e} y^{e} d \sigma d t-\int_{\Sigma_{-}} \xi_{-}^{e} y^{e} d \sigma d t
$$

where we have taken averages of the observed data against given functions $\xi_{0}, \xi_{+}$and $\xi_{-}$. Unfortunately, such a functional may be sensitive to the missing data. The idea is to introduce new unknowns $w_{0}^{e}, w_{+}^{e}$ and $w_{-}^{e}$ and define the sentinel in the form

$$
\begin{aligned}
& S^{e}(\lambda, \tau)=e \int_{\Sigma_{0}}\left(\xi_{0}^{e}+w_{0}^{e}\right) \frac{\partial y^{e}}{\partial \nu} d \sigma d t- \\
& -\int_{\Sigma_{+}}\left(\xi_{+}^{e}+w_{+}^{e}\right) y^{e} d \sigma d t-\int_{\Sigma_{-}}\left(\xi_{-}^{e}+w_{-}^{e}\right) y^{e} d \sigma d t
\end{aligned}
$$

We seek elements $w_{0}^{e}, w_{ \pm}^{e}$ such that $S^{e}$ satisfies the insensivity condition given below:

$$
\left(\partial S^{e} / \partial \tau_{0}\right)(0,0)=0,\left(\partial S^{e} / \partial \tau_{1}\right)(0,0)=0 \quad \forall \widehat{y_{0}^{e}}, \widehat{y_{1}^{e}} .
$$

It is natural to require the norm of $\left(w_{0}^{e}, w_{ \pm}^{e}\right)$ to be minimal so that the value of the sentinel is not far from the one given by (2.5). This condition is automatically satisfied by the very nature of H.U.M. employed to choose $\left(w_{0}^{e}, w_{ \pm}^{e}\right)$ (cf. $\left.\S 8.4\right)$.

Thus, in order to construct the sentinel $S^{e}$, we need to solve (2.2) for $y^{e}$ and study its trace on $\Sigma_{ \pm}$and its normal trace on $\Sigma_{0}$. This is the object of $\S 3-\S 7$. The task in $\S 8$ is to find $\left(w_{0}^{e}, w_{ \pm}^{e}\right)$ such that $(2.7)$ is satisfied 


\section{Non-homogeneous mixed problems - weak so- lutions}

The purpose of this section is to describe some results giving existence, uniqueness and estimates independent of $e$ to solutions to the initialboundary value problems of mixed type (2.2) in the case of cylindrical domains considered in $\S 2$. It is to be noted that we have a nonhomogeneous mixed Dirichlet-Neumann condition in (2.2). Several results concerning the pure Dirichlet and the pure Neumann problems are already available in the literature and we cite, for instance, Lions [6], Lasiecka, Lions \& Triggiani [3], Lasiecka \& Triggiani [4].

We will be primarily concerned with three types of solutions: regular solutions belonging to $C^{0}\left([0, T] ; H^{s}(\Omega)\right)$ for some $s>3 / 2$, finite energy solutions belonging to $C^{0}\left([0, T] ; H^{1}(\Omega)\right)$ and solutions in the space $C^{0}\left([0, T] ; L^{2}(\Omega)\right)$ which will be called weak solutions. In this section, we focus our attention to the properties of weak solutions. Our plan is to consider some properties of finite energy solutions with homogeneous boundary conditions and, by duality arguments, deduce some information on weak solutions.

\subsection{Homogeneous mixed problem}

Let us first introduce the function spaces

$$
\left\{\begin{array}{l}
H=L^{2}(\Omega), \quad V=\left\{v \in H^{1}(\Omega) ; v=0 \text { on } \Gamma_{0}\right\} \\
V^{e} \text { denotes the space } V \text { with the norm }\|v\|_{V^{e}}=\left\|\nabla_{e} v\right\|_{(H)^{3}} \\
\text { where } \nabla_{e} v=\left(\left(\partial v / \partial z_{1}\right),\left(\partial v / \partial z_{2}\right), e^{-1}\left(\partial v / \partial z_{3}\right)\right)
\end{array}\right.
$$

As usual, we identify $L^{2}(\Omega)$ with its dual and, in this case we have the dense inclusions:

$$
V^{e} \hookrightarrow H=H^{\prime} \hookrightarrow\left(V^{e}\right)^{\prime} .
$$

With these notations, let us consider solutions $\theta^{e}$ of finite energy to homogeneous initial-boundary value problem with mixed boundary condition:

$$
\left\{\begin{array}{l}
\square_{e} \theta^{e}=F^{e} \quad \text { in } Q \\
\theta^{e}=0 \text { on } \Sigma_{0} \text { and } e^{-1}\left(\partial \theta^{e} / \partial \nu\right)=0 \text { on } \Sigma_{ \pm} \\
\theta^{e}(0)=\theta_{0}^{e} \text { and }\left(\theta^{e}\right)^{\prime}(0)=\theta_{1}^{e}
\end{array}\right.
$$


where we take

$$
F^{e} \in L^{1}(0, T ; H), \quad \theta_{0}^{e} \in V^{e}, \quad \theta_{1}^{e} \in H
$$

We define the energy associated to problem (3.2):

$$
E^{e}\left(\theta^{e} ; t\right)=\frac{1}{2} \int_{\Omega}\left\{\left(\frac{\partial \theta^{e}}{\partial t}\right)^{2}+\left|\nabla_{e} \theta^{e}\right|^{2}\right\} d z .
$$

We recall the following result from Saint Jean Paulin \& Vanninathan [8]:

Theorem 3.1. Under the condition (3.3), there exists a unique solution $\theta^{e}$ satisfying (3.2) and

(3.5) $\theta^{e} \in C^{0}\left([0, T] ; V^{e}\right),\left(\theta^{e}\right)^{\prime} \in C^{0}([0, T] ; H),\left(\theta^{e}\right)^{\prime \prime} \in L^{1}\left(0, T ;\left(V^{e}\right)^{\prime}\right)$.

Moreover, for all multipliers $m_{k} \in W^{1, \infty}(\Omega), k=1,2,3$, the solution $\theta^{e}$ satisfies the following identity:

$$
\begin{aligned}
& \frac{1}{2} \int_{\Sigma_{0}}\left(m_{\alpha} \nu_{\alpha}\right)\left(\frac{\partial \theta^{e}}{\partial \nu}\right)^{2} d \sigma d t+ \\
& +\frac{1}{2} \int_{\Sigma_{+} \cup \Sigma_{-}}\left(m_{3} \nu_{3}\right)\left\{\left(\frac{\partial \theta^{e}}{\partial t}\right)^{2}-\frac{\partial \theta^{e}}{\partial z_{\alpha}} \frac{\partial \theta^{e}}{\partial z_{\alpha}}\right\} d z d t= \\
& =\left[\int_{\Omega} \frac{\partial \theta^{e}}{\partial t} m_{k} \frac{\partial \theta^{e}}{\partial z_{k}} d z\right]_{0}^{T}+\frac{1}{2} \int_{Q} \frac{\partial m_{k}}{\partial z_{k}}\left\{\left(\frac{\partial \theta^{e}}{\partial t}\right)^{2}-\left|\nabla_{e} \theta^{e}\right|^{2}\right\} d z d t+ \\
& +\int_{Q} \frac{\partial m_{k}}{\partial z_{\alpha}} \frac{\partial \theta^{e}}{\partial z_{\alpha}} \frac{\partial \theta^{e}}{\partial z_{k}} d z d t+e^{-2} \int_{Q} \frac{\partial m_{k}}{\partial z_{3}} \frac{\partial \theta^{e}}{\partial z_{3}} \frac{\partial \theta^{e}}{\partial z_{k}} d z d t- \\
& -\int_{Q}^{F^{e} m_{k}} \frac{\partial \theta^{e}}{\partial z_{k}} d z d t
\end{aligned}
$$

Further, there exists a constant $C>0$ such that

$$
\begin{aligned}
& E^{e}\left(\theta^{e} ; t\right)+\int_{\Sigma_{0}}\left(\frac{\partial \theta^{e}}{\partial \nu}\right)^{2} d \sigma d t+ \\
& +\left|\int_{\Sigma_{+}}\left\{\left(\frac{\partial \theta^{e}}{\partial t}\right)^{2}-\frac{\partial \theta^{e}}{\partial z_{\alpha}} \frac{\partial \theta^{e}}{\partial z_{\alpha}}\right\} d \sigma d t\right|+ \\
& +\left|\int_{\Sigma_{-}}\left\{\left(\frac{\partial \theta^{e}}{\partial t}\right)^{2}-\frac{\partial \theta^{e}}{\partial z_{\alpha}} \frac{\partial \theta^{e}}{\partial z_{\alpha}}\right\} d \sigma d t\right| \leq \\
& \leq C\left\{E^{e}\left(\theta^{e} ; 0\right)+\left(\int_{0}^{T}\left\|F^{e}(t)\right\|_{H} d t\right)^{2}\right\} .
\end{aligned}
$$


Here, and in the sequel, $C$ denotes a generic constant independent of the thickness parameter $e$.

In our study, we require certain other estimates also on the problem (3.2). Let us take

$$
F^{e}=\left(F_{1}^{e}\right)^{\prime}\left(\equiv \partial F_{1}^{e} / \partial t\right) \quad \text { with } F_{1}^{e} \in L^{1}\left(0, T ; V^{e}\right) .
$$

Theorem 3.2. We suppose (3.7) and take $\theta_{0}^{e}=0$ and $\theta_{1}^{e}=0$. Then the following estimates hold for solutions of (3.2):

$$
\left\{\begin{array}{l}
\left\|\nabla_{e} \theta^{e}\right\|_{L^{\infty}(0, T ; H)}+\left\|\left(\theta^{e}\right)^{\prime}\right\|_{L^{\infty}(0, T ; H)} \leq C\left\|\nabla_{e} F_{1}^{e}\right\|_{L^{1}(0, T ; H)} \\
\left\|\nabla \theta^{e}\right\|_{L^{\infty}(0, T ; H)} \leq C\left\|\nabla F_{1}^{e}\right\|_{L^{1}(0, T ; H)} \\
\left\|\left(\partial \theta^{e} / \partial \nu\right)\right\|_{L^{2}\left(\Sigma_{0}\right)} \leq C\left\|\nabla_{e} F_{1}^{e}\right\|_{L^{1}(0, T ; H)}
\end{array}\right.
$$

Proof. We sketch the proof, following the ideas of Lions [6] who treated the Dirichlet problem. By density arguments, we can assume $F_{1}^{e}$ is smooth, $F_{1}^{e}(0)=0$ and $F_{1}^{e}(T)=0$. We introduce $w^{e}$ the solution of:

$$
\left\{\begin{array}{l}
\square_{e} w^{e}=F_{1}^{e} \quad \text { in } Q \\
w^{e}=0 \text { on } \Sigma_{0} \text { and } e^{-1}\left(\partial w^{e} / \partial \nu\right)=0 \text { on } \Sigma_{ \pm}, \\
w^{e}(0)=\left(w^{e}\right)^{\prime}(0)=0,
\end{array}\right.
$$

and note that $\theta^{e}=\left(w^{e}\right)^{\prime}$. Multiplying (3.9) by $-\Delta_{e}\left(w^{e}\right)^{\prime}$ and integrating by parts, we get

$$
\left\|\nabla_{e}\left(w^{e}\right)^{\prime}\right\|_{L^{\infty}(0, T ; H)}+\left\|\Delta_{e} w^{e}\right\|_{L^{\infty}(0, T ; H)} \leq C\left\|\nabla_{e} F_{1}^{e}\right\|_{L^{1}(0, T ; H)} .
$$

This implies immediately that

$$
\left\|\left(\theta^{e}\right)^{\prime}(T)\right\|_{H} \leq C\left\|\nabla_{e} F_{1}^{e}\right\|_{L^{1}(0, T ; H)} .
$$

Since $T$ is arbitrary, we have the first inequality in (3.8).

To prove the second inequality in (3.8), we multiply (3.9) by $-\Delta\left(w^{e}\right)^{\prime}$ and integrate by parts. We obtain

$$
\begin{aligned}
& e^{-1}\left\|\left(\partial^{2} w^{e} / \partial z_{3}^{2}\right)\right\|_{L^{\infty}(0, T ; H)}+\left\|\left(\partial^{2} w^{e} / \partial z_{\alpha} \partial z_{3}\right)\right\|_{L^{\infty}(0, T ; H)}+ \\
& +e^{-1}\left\|\left(\partial^{2} w^{e} / \partial z_{\alpha} \partial z_{\alpha}\right)\right\|_{L^{\infty}(0, T ; H)}+\left\|\nabla\left(w^{e}\right)^{\prime}\right\|_{L^{\infty}(0, T ; H)} \leq \\
& \leq C\left\|\nabla F_{1}^{e}\right\|_{L^{1}(0, T ; H)} .
\end{aligned}
$$


To prove the third inequality in (3.8) we multiply (3.2) by $m_{k}\left(\partial \theta^{e} / \partial z_{k}\right)$ with $m_{k}$ in $W^{1, \infty}(\Omega)$. We arrive at the following identity:

$$
\begin{aligned}
& \frac{1}{2} \int_{\Sigma_{0}}\left(m_{\alpha} \nu_{\alpha}\right)\left(\frac{\partial \theta^{e}}{\partial \nu}\right)^{2} d \sigma d t+ \\
& +\frac{1}{2} \int_{\Sigma_{+} \cup \Sigma_{-}}\left(m_{3} \nu_{3}\right)\left\{\left(\frac{\partial \theta^{e}}{\partial t}\right)^{2}-\frac{\partial \theta^{e}}{\partial z_{\alpha}} \frac{\partial \theta^{e}}{\partial z_{\alpha}}\right\} d \sigma d t= \\
& =\int_{\Omega}\left(\frac{\partial \theta^{e}}{\partial t}\right)(T) m_{k} \frac{\partial \theta^{e}}{\partial z_{k}}(T) d z+ \\
& +\frac{1}{2} \int_{Q} \frac{\partial m_{k}}{\partial z_{k}}\left\{\left|\Delta_{e} w^{e}\right|^{2}-\left|\nabla_{e} \theta^{e}\right|^{2}\right\} d z d t+ \\
& +\int_{Q} \frac{\partial \theta^{e}}{\partial z_{\alpha}} \frac{\partial m_{k}}{\partial z_{\alpha}} \frac{\partial \theta^{e}}{\partial z_{k}} d z d t+e^{-2} \int_{Q} \frac{\partial \theta^{e}}{\partial z_{k}} \frac{\partial m_{k}}{\partial z_{3}} \frac{\partial \theta^{e}}{\partial z_{k}} d z d t- \\
& -\int_{Q}^{Q} \frac{\partial F_{1}^{e}}{\partial z_{k}} m_{k} \Delta_{e} w^{e} d z d t-\frac{1}{2} \int_{\Sigma_{+} \cup \Sigma_{-}}^{Q}\left(m_{3} \nu_{3}\right)\left(F_{1}^{e}\right)^{2} d \sigma d t+ \\
& +\int_{\Sigma_{+} \cup \Sigma_{-}}^{Q}\left(m_{3} \nu_{3}\right) F_{1}^{e}\left(\frac{\partial \theta^{e}}{\partial t}\right) d \sigma d t
\end{aligned}
$$

Taking into account the cylindrical geometry of our domain $\Omega$, we choose the multipliers $m_{k}, k=1,2,3$ which were introduced in Saint Jean Paulin \& Vanninathan [8]:

(3.13) $m_{1}, m_{2}$ independent of $z_{3}, \quad m_{\alpha}=\nu_{\alpha}$ on $\Gamma_{0}$ and $m_{3}=0$.

Using these multipliers in (3.12), we can easily complete the proof of (3.8).

As shown by Theorem 3.1, the trace on $\Sigma_{+}$of solutions $\theta^{e}$ of (3.2) (with $\left.F^{e} \in L^{1}(0, T ; H)\right)$ satisfies the inequality (3.6). Let us introduce the spaces $W_{ \pm}^{e}$ consisting of traces on $\Sigma_{ \pm}$of solutions $\theta^{e}$ of (3.2). We provide $W_{ \pm}^{e}$ with the following "quotient norm":

$$
\begin{aligned}
\left\|w_{ \pm}^{e}\right\|_{W_{ \pm}^{e}}= & \inf \left\{\left\|F^{e}\right\|_{L^{1}(0, T ; H)}+\left\|\theta_{0}^{e}\right\|_{V^{e}}+\left\|\theta_{1}^{e}\right\|_{H} \mid \theta^{e}\right. \text { is associated } \\
& \text { to } \left.\left(F^{e}, \theta_{0}^{e}, \theta_{1}^{e}\right) \text { via }(3.2) \text { and }\left.\theta^{e}\right|_{\Sigma_{ \pm}}=w_{ \pm}^{e}\right\} .
\end{aligned}
$$

The dual spaces are denoted by $\left(W_{ \pm}^{e}\right)^{\prime}$. Since $\theta^{e} \in C^{0}\left([0, T] ; V^{e}\right)$ (cf $(3.5))$, it follows from the trace results on $H^{1}(\Omega)$ that we have the inclusions $W_{ \pm}^{e} \subseteq C^{0}\left([0, T] ; H^{1 / 2}\left(\Gamma_{ \pm}\right)\right)$. 


\subsection{Existence of weak solutions}

We seek weak solutions $\psi^{e}$ satisfying

$$
\left\{\begin{array}{l}
\square_{e} \psi^{e}=f^{e} \quad \text { in } Q, \\
\psi^{e}=g_{0}^{e} \text { on } \Sigma_{0} \text { and } e^{-1}\left(\partial \psi^{e} / \partial \nu\right)=g_{ \pm}^{e} \quad \text { on } \Sigma_{ \pm}, \\
\psi^{e}(0)=\psi_{0}^{e} \text { and }\left(\psi^{e}\right)^{\prime}(0)=\psi_{1}^{e} \text { in } \Omega .
\end{array}\right.
$$

The following result proves not only existence and uniqueness of weak solutions of (3.14) but also specifies the precise sense in which (3.14) has to be understood.

Theorem 3.3. We make the hypotheses that

$$
\begin{aligned}
& f^{e} \in L^{1}\left(0, T ;\left(V^{e}\right)^{\prime}\right), \psi_{0}^{e} \in H, \psi_{1}^{e} \in\left(V^{e}\right)^{\prime}, \\
& g_{0}^{e} \in L^{2}\left(\Sigma_{0}\right), g_{ \pm}^{e} \in\left(V_{ \pm}^{e}\right)^{\prime},
\end{aligned}
$$

where we take $V_{ \pm}^{e}=W_{ \pm}^{e}$ (or) $V_{ \pm}^{e}=L^{2}\left(0, T ; H^{1 / 2}\left(\Gamma_{ \pm}\right)\right)$.

Then, there exists a unique weak solution $\psi^{e}$ of (3.14) in the following sense:

$$
\psi^{e} \in C^{0}([0, T] ; H),
$$

$$
\begin{aligned}
& L^{1}(0, T ; H)<F^{e}, \psi^{e}>_{L^{\infty}(0, T ; H)}= \\
& =L_{L^{1}\left(0, T ;\left(V^{e}\right)^{\prime}\right)<f^{e}, \theta^{e}>_{L^{\infty}\left(0, T ; V^{e}\right)}+\left(V^{e}\right)^{\prime}}<\psi_{1}^{e}, \theta^{e}(0)>_{V^{e}-} \\
& { }_{H}<\psi_{0}^{e},\left(\theta^{e}\right)^{\prime}(0)>_{H}-L_{L^{2}\left(\Sigma_{0}\right)}<g_{0}^{e}, \partial \theta^{e} / \partial \nu>_{L^{2}\left(\Sigma_{0}\right)}+ \\
& +{ }_{\left(V_{+}^{e}\right)^{\prime}}<e^{-1} g_{+}^{e}, \theta^{e}>_{V_{+}^{e}}+\left(V_{-}^{e}\right)^{\prime}<e^{-1} g_{-}^{e}, \theta^{e}>_{V_{-}^{e}},
\end{aligned}
$$

for all $F^{e} \in L^{1}(0, T ; H)$ and for all finite energy solutions $\theta^{e}$ satisfying the following backward mixed homogeneous initial-boundary value problem:

$$
\left\{\begin{array}{l}
\square_{e} \theta^{e}=F^{e} \text { in } Q, \\
\theta^{e}=0 \text { on } \Sigma_{0} \quad \text { and } \quad e^{-1}\left(\partial \theta^{e} / \partial \nu\right)=0 \quad \text { on } \Sigma_{ \pm}, \\
\theta^{e}(T)=\left(\theta^{e}\right)^{\prime}(T)=0 \text { in } \Omega .
\end{array}\right.
$$

Moreover, we have the following estimate:

$$
\left\{\begin{array}{c}
\left\|\psi^{e}\right\|_{L^{\infty}(0, T ; H)} \leq C R_{1}\left(f^{e}, \psi_{0}^{e}, \psi_{1}^{e}, g_{0}^{e}, g_{+}^{e}, g_{-}^{e}\right) \quad \text { with } \\
R_{1}\left(f^{e}, \psi_{0}^{e}, \psi_{1}^{e}, g_{0}^{e}, g_{+}^{e}, g_{-}^{e}\right) \equiv\left\|f^{e}\right\|_{L^{1}\left(0, T ;\left(V^{e}\right)^{\prime}\right)}+ \\
+\left\|\psi_{0}^{e}\right\|_{H}+\left\|\psi_{1}^{e}\right\|_{\left(V^{e}\right)^{\prime}+} \\
+\left\|g_{0}^{e}\right\|_{L^{2}\left(\Sigma_{0}\right)}+e^{-1}\left\|g_{+}^{e}\right\|_{\left(V_{+}^{e}\right)^{\prime}}+ \\
+e^{-1}\left\|g_{-}^{e}\right\|_{\left(V_{-}^{e}\right)^{\prime}}
\end{array}\right.
$$


Proof. We follow the transposition technique to solve (3.14). Indeed the formulation (3.17) is obtained by multiplying (3.14) by $\theta^{e}$ solution of (3.18) and applying Green's formula, assuming sufficient regularity of $\theta^{e}$ and $\psi^{e}$.

To finish the proof, it suffices to consider the right-hand side of (3.17) as a linear map of $F^{e} \in L^{1}(0, T ; H)$. Thanks to our assumptions and the inequality (3.6) for the backward problem, the above map is continuous and hence given by a unique $\psi^{e} \in L^{\infty}(0, T ; H)$ satisfying the estimate (3.19). The passage from the regularity $\psi^{e} \in L^{\infty}(0, T ; H)$ to $\psi^{e} \in C^{0}([0, T] ; H)$ is done by the usual techniques (adapt for instance Lions [6], tome 1 , p. 46 to our case).

Corollary 3.4. The spaces $W_{ \pm}^{e}$ are dense in $L^{2}\left(\Sigma_{ \pm}\right)$respectively.

Proof. Let $g_{ \pm}^{e} \in L^{2}\left(\Sigma_{ \pm}\right)$be such that ${ }_{L^{2}\left(\Sigma_{ \pm}\right)}<g_{ \pm}^{e}, \theta^{e}>_{L^{2}\left(\Sigma_{ \pm}\right)}=0$ for all solutions $\theta^{e}$ of (3.2), and in particular solutions of (3.18). By Theorem 3.3, we know that there exists a unique weak solution $\psi^{e} \in L^{\infty}(0, T ; H)$ of (3.14) where we take $g_{ \pm}^{e} \in L^{2}\left(\Sigma_{ \pm}\right) \subset L^{2}\left(0, T ; H^{-1 / 2}\left(\Gamma_{ \pm}\right)\right)$and $f^{e}=0, g_{0}^{e}=0, \psi_{0}^{e}=0, \psi_{1}^{e}=0$. If we apply (3.17), we obtain

$$
\begin{aligned}
& L^{1}(0, T ; H)<F^{e}, \psi^{e}>_{L^{\infty}(0, T ; H)}= \\
& ={ }_{L^{2}\left(\Sigma_{+}\right)}<e^{-1} g_{+}^{e}, \theta^{e}>_{L^{2}\left(\Sigma_{+}\right)}+{ }_{L^{2}\left(\Sigma_{-}\right)}<e^{-1} g_{-}^{e}, \theta^{e}>_{L^{2}\left(\Sigma_{-}\right)}=0 .
\end{aligned}
$$

Since $F^{e}$ is arbitrary, we conclude that $\psi^{e}=0$ and hence that $g_{ \pm}^{e}=0$.

\subsection{Additional estimate on weak solutions}

In this paragraph, we derive an estimate on $\left(\psi^{e}\right)^{\prime}$ in the space $L^{\infty}\left(0, T ;\left(V^{e}\right)^{\prime}\right)$ where $\psi^{e}$ is the weak solution of (3.14).

Theorem 3.5. Under the assumptions of Theorem 3.3, $\left(\psi^{e}\right)^{\prime} \in$ $C^{0}\left([0, T] ;\left(V^{e}\right)^{\prime}\right)$ and

$$
\left\|\left(\psi^{e}\right)^{\prime}\right\|_{L^{\infty}\left(0, T ;\left(V^{e}\right)^{\prime}\right)} \leq C R_{1}\left(f^{e}, \psi_{0}^{e}, \psi_{1}^{e}, g_{0}^{e}, g_{+}^{e}, g_{-}^{e}\right) .
$$


Proof. As in the proof of the previous theorem, we consider the linear functional $F^{e} \rightarrow L\left(F^{e}\right)$ defined by the right-hand side of (3.17) with the choice that $F^{e}=\left(F_{1}^{e}\right)^{\prime}$ where $F_{1}^{e} \in L^{1}\left(0, T ; V^{e}\right)$. Applying the estimates (3.8), we deduce that (see Lions [6] in the Dirichlet case)

$$
\left|L\left(F^{e}\right)\right| \leq C\left\|F^{e}\right\|_{W^{-1,1}\left(0, T ; V^{e}\right)} R_{1}\left(f^{e}, \psi_{0}^{e}, \psi_{1}^{e}, g_{0}^{e}, g_{+}^{e}, g_{-}^{e}\right) .
$$

This easily implies that $\psi^{e} \in W^{1, \infty}\left(0, T ;\left(V^{e}\right)^{\prime}\right)$ and (3.20) holds.

\section{Non-homogeneous mixed problems - finite en- ergy solutions}

We concentrate our efforts in this section in obtaining existence, uniqueness and estimates of finite energy solutions to the system

$$
\left\{\begin{array}{l}
\square_{e} \theta^{e}=F^{e} \text { in } Q, \\
\theta^{e}=h_{0}^{e} \text { on } \Sigma_{0} \text { and } e^{-1}\left(\partial \theta^{e} / \partial \nu\right)=h_{ \pm}^{e} \quad \text { on } \Sigma_{ \pm} \\
\theta^{e}(0)=\theta_{0}^{e} \text { and }\left(\theta^{e}\right)^{\prime}(0)=\theta_{1}^{e} \quad \text { in } \Omega .
\end{array}\right.
$$

It will be convenient to decompose the solution into two parts: $\theta^{e}=$ $\bar{\theta}^{e}+\left(\theta^{e}-\bar{\theta}^{e}\right)$ where $\bar{\theta}^{e}$ and $\theta^{e}-\bar{\theta}^{e}$ are solutions of the following systems respectively:

$$
\left\{\begin{array}{l}
\square_{e} \bar{\theta}^{e}=0 \quad \text { in } Q \\
\bar{\theta}^{e}=h_{0}^{e} \text { on } \Sigma_{0} \quad \text { and } e^{-1}\left(\partial \bar{\theta}^{e} / \partial \nu\right)=h_{ \pm}^{e} \quad \text { on } \Sigma_{ \pm} \\
\bar{\theta}^{e}(0)=\theta_{0}^{e} \text { and } \quad\left(\bar{\theta}^{e}\right)^{\prime}(0)=\theta_{1}^{e} \quad \text { in } \Omega
\end{array}\right.
$$

$$
\left\{\begin{array}{l}
\square_{e}\left(\theta^{e}-\bar{\theta}^{e}\right)=F^{e} \quad \text { in } Q \\
\left(\theta^{e}-\bar{\theta}^{e}\right)=0 \text { on } \Sigma_{0} \text { and } e^{-1}(\partial / \partial \nu)\left(\theta^{e}-\bar{\theta}^{e}\right)=0 \text { on } \Sigma_{ \pm} \\
\left(\theta^{e}-\bar{\theta}^{e}\right)(0)=\left(\theta^{e}-\bar{\theta}^{e}\right)^{\prime}(0)=0 \text { in } \Omega
\end{array}\right.
$$

A simple application of Theorem 3.1 yields

$$
\left\|\nabla_{e}\left(\theta^{e}-\bar{\theta}^{e}\right)\right\|_{L^{\infty}(0, T ; H)^{3}}+\left\|\left(\theta^{e}-\bar{\theta}^{e}\right)^{\prime}\right\|_{L^{\infty}(0, T ; H)} \leq C\left\|F^{e}\right\|_{L^{1}(0, T ; H)} .
$$

It remains to study problem (4.2) and exhibit solutions of finite energy for (4.2). This is the object of the following paragraphs. 


\subsection{First results on problem (4.2)}

To obtain finite energy solutions of (4.2), we need to follow a different strategy. The technique adopted in the result below yields only an estimate on $\left(\bar{\theta}^{e}\right)^{\prime}$ in $L^{\infty}(0, T ; H)$. To get an estimate on $\bar{\theta}^{e}$ in $L^{\infty}\left(0, T ; H^{1}(\Omega)\right)$, we have to follow yet another method which will be taken up in the next paragraph.

Theorem 4.1. We assume that

$$
\left\{\begin{array}{l}
\theta_{0}^{e} \in H^{1}(\Omega), \partial \theta_{0}^{e} / \partial \nu \in H^{-1 / 2}\left(\Gamma_{ \pm}\right), \theta_{1}^{e} \in H \\
h_{0}^{e} \in H^{1}\left(0, T ; L^{2}\left(\Gamma_{0}\right)\right) \cap C^{0}\left([0, T] ; H^{1 / 2}\left(\Gamma_{0}\right)\right) \\
h_{ \pm}^{e} \in C^{0}\left([0, T] ; H^{-1 / 2}\left(\Gamma_{ \pm}\right)\right),\left(h_{ \pm}^{e}\right)^{\prime} \in\left(W_{ \pm}^{e}\right)^{\prime}
\end{array}\right.
$$

In addition, we assume the following compatibility conditions:

$$
\left.h_{0}^{e}\right|_{t=0}=\theta_{0}^{e} \quad \text { on } \Gamma_{0} \quad \text { and }\left.\quad h_{ \pm}^{e}\right|_{t=0}=e^{-1}\left(\partial \theta_{0}^{e} / \partial \nu\right) \quad \text { on } \Gamma_{ \pm} .
$$

Then, there exists a unique solution $\bar{\theta}^{e}$ satisfying (4.2) with the following regularity:

$$
\bar{\theta}^{e} \in C^{1}([0, T] ; H),\left(\bar{\theta}^{e}\right)^{\prime \prime} \in C^{0}\left([0, T] ;\left(V^{e}\right)^{\prime}\right) .
$$

Further, we have the estimates

$$
\left\{\begin{array}{c}
\left\|\left(\bar{\theta}^{e}\right)^{\prime}\right\|_{L^{\infty}(0, T ; H)}+ \\
\quad\left\|\left(\bar{\theta}^{e}\right)^{\prime \prime}\right\|_{L^{\infty}\left(0, T ;\left(V^{e}\right)^{\prime}\right)} \leq \\
\text { with } \left.R_{2}\left(\theta_{0}^{e}, \theta_{1}^{e}, h_{0}^{e}, h_{+}^{e}, h_{-}^{e}\right) \equiv \| \theta_{1}^{e}, h_{0}^{e}, h_{+}^{e}, h_{-}^{e}\right) \\
+\left\|\Delta_{e} \theta_{0}^{e}\right\|_{(V e)^{\prime}}+\left\|\left(h_{0}^{e}\right)^{\prime}\right\|_{L^{2}\left(\Sigma_{0}\right)}+ \\
+e^{-1}\left\|\left(h_{+}^{e}\right)^{\prime}\right\|_{\left(W_{+}^{e}\right)^{\prime}}+e^{-1}\left\|\left(h_{-}^{e}\right)^{\prime}\right\|_{\left(W_{-}^{e}\right)^{\prime}}
\end{array}\right.
$$

Proof. We set $\widetilde{\theta}^{e}=\left(\bar{\theta}^{e}\right)^{\prime}$ and note that $\widetilde{\theta}^{e}$ formally satisfies the following system:

$$
\left\{\begin{array}{l}
\square_{e} \widetilde{\theta}^{e}=0 \quad \text { in } Q \\
\widetilde{\theta}^{e}=\left(h_{0}^{e}\right)^{\prime} \quad \text { on } \Sigma_{0} \quad \text { and } \quad e^{-1}\left(\partial \widetilde{\theta}^{e} / \partial \nu\right)=\left(h_{ \pm}^{e}\right)^{\prime} \quad \text { on } \Sigma_{ \pm} \\
\widetilde{\theta}^{e}(0)=\theta_{1}^{e} \quad \text { and }\left(\widetilde{\theta}^{e}\right)^{\prime}(0)=\Delta_{e} \theta_{0}^{e} \quad \text { in } \Omega
\end{array}\right.
$$

It is in the verification of (4.9) in the sense of duality (cf. (3.17)) that we use the compatibility conditions (4.6). 
It is important to note that $\Delta_{e} \theta_{0}^{e} \in\left(V^{e}\right)^{\prime}$ and its action is indeed defined by

$$
\begin{aligned}
& \left(V^{e}\right)^{\prime}<\Delta_{e} \theta_{0}^{e}, v>_{V^{e}}=-\int_{\Omega}\left\{\frac{\partial \theta_{0}^{e}}{\partial z_{\alpha}} \frac{\partial v}{\partial z_{\alpha}}+e^{-2} \frac{\partial \theta_{0}^{e}}{\partial z_{3}} \frac{\partial v}{\partial z_{3}}\right\} d z+ \\
& +e_{H^{-1 / 2}\left(\Gamma_{+}\right)}^{-2}<\frac{\partial \theta_{0}^{e}}{\partial \nu}, v>_{H^{1 / 2}\left(\Gamma_{+}\right)}+ \\
& +e_{H^{-1 / 2}\left(\Gamma_{-}\right)}^{-2}<\frac{\partial \theta_{0}^{e}}{\partial \nu}, v>_{H^{1 / 2}\left(\Gamma_{-}\right)},
\end{aligned}
$$

for all $v \in V^{e}$. We now apply Theorems 3.3, 3.5 to problem (4.9) and conclude.

To show that $\bar{\theta}^{e}$ has finite energy, it remains to prove that $\bar{\theta}^{e} \in$ $C^{0}\left([0, T] ; H^{1}(\Omega)\right)$. To this end, we use the equation in (4.2). More precisely, we have the following stationary problem in which $t$ plays the rôle of a parameter

$$
\left\{\begin{array}{l}
-\Delta_{e} \bar{\theta}^{e}(t)=-\left(\bar{\theta}^{e}\right)^{\prime \prime}(t) \in\left(V^{e}\right)^{\prime} \quad \text { in } \Omega \\
\bar{\theta}^{e}(t)=h_{0}^{e}(t) \quad \text { on } \Gamma_{0}, e^{-1}\left(\partial \theta^{e} / \partial \nu\right)(t)=h_{ \pm}^{e}(t) \quad \text { on } \Gamma_{ \pm} .
\end{array}\right.
$$

\subsection{Stationary problems of mixed boundary conditions}

In the last paragraph, we were led to study stationary problems of the following form:

$$
\left\{\begin{array}{l}
-\Delta_{e} \psi^{e}=G^{e} \quad \text { in } \Omega \\
\psi^{e}=h_{0}^{e} \text { on } \Gamma_{0} \quad \text { and } \quad e^{-1}\left(\partial \psi^{e} / \partial \nu\right)=h_{ \pm}^{e} \quad \text { on } \Gamma_{ \pm}
\end{array}\right.
$$

It is classically known that there exists a unique solution $\psi^{e} \in H^{1}(\Omega)$ if $G^{e} \in\left(V^{e}\right)^{\prime}, h_{0}^{e} \in H^{1 / 2}\left(\Gamma_{0}\right)$ and $h_{ \pm}^{e} \in H^{-1 / 2}\left(\Gamma_{ \pm}\right)$. However, in order to have estimates on $\psi^{e}$ uniform with respect to $e$, we need to assume more regularity on the data. This is the purpose of this paragraph.

We decompose the solution into two parts $\psi^{e}=\bar{\psi}^{e}+\left(\psi^{e}-\bar{\psi}^{e}\right)$ where $\bar{\psi}^{e}$ satisfies

$$
\left\{\begin{array}{l}
-\Delta_{e} \bar{\psi}^{e}=0 \quad \text { in } \Omega \\
\bar{\psi}^{e}=h_{0}^{e} \quad \text { on } \Gamma_{0} \text { and } e^{-1}\left(\partial \bar{\psi}^{e} / \partial \nu\right)=h_{ \pm}^{e} \quad \text { on } \Gamma_{ \pm} .
\end{array}\right.
$$


Clearly $\left(\psi^{e}-\bar{\psi}^{e}\right)$ satisfies (4.12) with $h_{0}^{e}=h_{ \pm}^{e}=0$. It is easy to deduce the required energy estimate on $\left(\psi^{e}-\bar{\psi}^{e}\right)$ from the variational formulation of problem (4.12) with homogeneous boundary conditions. We obtain

$$
\left\|\nabla_{e}\left(\psi^{e}-\bar{\psi}^{e}\right)\right\|_{H^{3}} \leq\left\|G^{e}\right\|_{\left(V^{e}\right)^{\prime}} .
$$

Now, it remains to study the problem (4.13). We will need some additional hypotheses on $h_{0}^{e}$ and $h_{ \pm}^{e}$ in our analysis. To introduce these, let us denote by $\tau^{1}(z), \tau^{2}(z)$ a piece-wise smooth selection of orthonormal tangent vectors at boundary points $z \in \partial \Omega$ such that their components satisfy

$$
\left\{\begin{array}{l}
\tau^{1}=(0,0,1) \quad \text { and } \quad\left(\tau^{2}\right)_{3}=0 \quad \text { on } \Gamma_{0} \\
\tau^{1}=(1,0,0) \quad \text { and } \quad \tau^{2}=(0,1,0) \quad \text { on } \Gamma_{ \pm} .
\end{array}\right.
$$

We can express the gradient of a function $\chi$ as follows:

$$
\partial \chi / \partial z_{k}=\nu_{k}(\partial \chi / \partial \nu)+\sum_{j=1}^{2}\left(\tau^{j}\right)_{k}\left(\partial \chi / \partial \tau^{j}\right) \quad k=1,2,3
$$

where $\partial \chi / \partial \tau^{j}$ are tangential derivatives of $\chi$ on the boundary. We set

$$
\sigma_{k} \chi=\sum_{j=1}^{2}\left(\tau^{j}\right)_{k}\left(\partial \chi / \partial \tau^{j}\right) \text { and } \nabla_{\sigma} \chi=\left\{\sigma_{k} \chi\right\}_{k=1}^{3} .
$$

We are now in a position to state our next result.

Theorem 4.2. We suppose that $h_{0}^{e} \in H^{1}\left(\Gamma_{0}\right)$ and $h_{ \pm}^{e} \in H^{-1 / 2}\left(\Gamma_{ \pm}\right)$. Then we have the estimate satisfied by the solution $\bar{\psi}^{e}$ of (4.13):

$$
\left\{\begin{aligned}
& \int_{\Omega}\left|\nabla_{e} \bar{\psi}^{e}\right|^{2} d z \leq C R_{3}\left(h_{0}^{e}, h_{+}^{e}, h_{-}^{e}\right) \text { with } \\
& R_{3}\left(h_{0}^{e}, h_{+}^{e}, h_{-}^{e}\right) \equiv \int_{\Gamma_{0}}\left\{\left|\nabla_{\sigma} h_{0}^{e}\right|^{2}+e^{-2} \frac{\partial h_{0}^{e}}{\partial z_{3}}+\left|h_{0}^{e}\right|^{2}\right\} d \sigma+ \\
&+e^{-2}\left\|h_{+}^{e}\right\|_{H^{-1 / 2}\left(\Gamma_{+}\right)}^{2}+e^{-2}\left\|h_{-}^{e}\right\|_{H^{-1 / 2}\left(\Gamma_{-}\right)}^{2}
\end{aligned}\right.
$$

Proof. We decompose $\bar{\psi}^{e}$ as follows: $\bar{\psi}^{e}=\xi^{e}+\left(\bar{\psi}^{e}-\xi^{e}\right)$ where $\xi^{e}$ is the solution of

$$
\left\{\begin{array}{l}
-\Delta_{e} \xi^{e}=0 \quad \text { in } \Omega, \\
\xi^{e}=h_{0}^{e} \quad \text { on } \Gamma_{0} \quad \text { and } \quad e^{-1}\left(\partial \xi^{e} / \partial \nu\right)=0 \quad \text { on } \Gamma_{ \pm} .
\end{array}\right.
$$


Then $\left(\bar{\psi}^{e}-\xi^{e}\right)$ satisfies clearly (4.13) with $h_{0}^{e}=0$. From the variational formulation of Problem (4.13) with homogeneous boundary conditions on $\Gamma_{0}$, we deduce, using Poincaré and trace inequalities, that, for $e<1$,

$$
\begin{aligned}
& \int_{\Omega}\left|\nabla_{e}\left(\bar{\psi}^{e}-\xi^{e}\right)\right|^{2} d z \leq \\
& \leq C\left\{e^{-2}\left\|h_{+}^{e}\right\|_{H^{-1 / 2}\left(\Gamma_{+}\right)}^{2}+e^{-2}\left\|h_{-}^{e}\right\|_{H^{-1 / 2}\left(\Gamma_{-}\right)}^{2}\right\} .
\end{aligned}
$$

Thus, we are reduced to derive the required estimate for the problem (4.18). As a first step towards this goal, let us multiply (4.18) by $\xi^{e}$. We obtain

$$
\int_{\Omega}\left|\nabla_{e} \xi^{e}\right|^{2} d z=\int_{\Gamma_{0}} h_{0}^{e} \frac{\partial \xi^{e}}{\partial \nu} d \sigma \leq\left\|h_{0}^{e}\right\|_{L^{2}\left(\Gamma_{0}\right)}\left\|\partial \xi^{e} / \partial \nu\right\|_{L^{2}\left(\Gamma_{0}\right)} .
$$

To estimate $\partial \xi^{e} / \partial \nu$ in $L^{2}\left(\Gamma_{0}\right)$, we multiply (4.18) by $m_{k}\left(\partial \xi^{e} / \partial z_{k}\right)$ where $m_{k} \in W^{1, \infty}(\Omega)$. After some computation, we arrive at

$$
\left\{\begin{array}{l}
\frac{1}{2} \int_{\Gamma_{0}}\left(m_{\beta} \nu_{\beta}\right)\left(\frac{\partial \xi^{e}}{\partial \nu}\right)^{2} d \sigma=\int_{\Omega} \frac{\partial \xi^{e}}{\partial z_{\alpha}} \frac{\partial m_{k}}{\partial z_{\alpha}} \frac{\partial \xi^{e}}{\partial z_{k}} d z- \\
-\frac{1}{2} \int_{\Omega} \frac{\partial m_{k}}{\partial z_{k}}\left|\nabla_{e} \xi^{e}\right|^{2} d z+ \\
+e^{-2} \int_{\Omega} \frac{\partial \xi^{e}}{\partial z_{3}} \frac{\partial m_{k}}{\partial z_{3}} \frac{\partial \xi^{e}}{\partial z_{k}} d z- \\
-\int_{\Gamma_{0}} \frac{\partial \xi^{e}}{\partial \nu}\left\{m_{\beta} \sigma_{\beta} h_{0}^{e}+m_{3} \frac{\partial h_{0}^{e}}{\partial z_{3}}\right\} d \sigma+ \\
+\frac{1}{2} \int_{\Gamma_{0}}\left(m_{\beta} \nu_{\beta}\right)\left\{\left|\nabla_{\sigma} h_{0}^{e}\right|^{2}+e^{-2}\left(\frac{\partial h_{0}^{e}}{\partial z_{3}}\right)^{2}\right\} d \sigma+ \\
+\frac{1}{2} \int_{\Gamma_{+} \cup \Gamma_{-}}\left(m_{3} \nu_{3}\right) \frac{\partial \xi^{e}}{\partial z_{\alpha}} \frac{\partial \xi^{e}}{\partial z_{\alpha}} d \sigma .
\end{array}\right.
$$

Let us now use the choice of multipliers defined by (3.13). We get

$$
\left\{\begin{array}{l}
\frac{1}{2} \int_{\Gamma_{0}}\left(\frac{\partial \xi^{e}}{\partial \nu}\right)^{2} d \sigma=\int_{\Omega} \frac{\partial \xi^{e}}{\partial z_{\alpha}} \frac{\partial m_{\beta}}{\partial z_{\alpha}} \frac{\partial \xi^{e}}{\partial z_{\beta}} d z- \\
-\frac{1}{2} \int_{\Omega} \frac{\partial m_{\beta}}{\partial z_{\beta}}\left|\nabla_{e} \xi^{e}\right|^{2} d z+ \\
+\frac{1}{2} \int_{\Gamma_{0}}\left\{\left|\nabla_{\sigma} h_{0}^{e}\right|^{2}+e^{-2}\left(\frac{\partial h_{0}^{e}}{\partial z_{3}}\right)^{2}\right\} d \sigma \\
\leq C\|m\|_{W^{1, \infty}(\Omega)^{2}}\left\|\nabla_{e} \xi^{e}\right\|_{H^{3}}^{2}+ \\
+\frac{1}{2} \int_{\Gamma_{0}}\left\{\left|\nabla_{\sigma} h_{0}^{e}\right|^{2}+e^{-2}\left(\frac{\partial h_{0}^{e}}{\partial z_{3}}\right)^{2}\right\} d \sigma
\end{array}\right.
$$


Combining (4.20) and (4.22), it is an easy matter to deduce

$$
\begin{aligned}
\int_{\Gamma_{0}}\left(\frac{\partial \xi^{e}}{\partial \nu}\right)^{2} d \sigma & +\int_{\Omega}\left|\nabla_{e} \xi^{e}\right|^{2} d z \leq \\
& \leq C \int_{\Gamma_{0}}\left\{\left|\nabla_{\sigma} h_{0}^{e}\right|^{2}+e^{-2}\left(\frac{\partial h_{0}^{e}}{\partial z_{3}}\right)^{2}+\left|h_{0}^{e}\right|^{2}\right\} d \sigma .
\end{aligned}
$$

Combining (4.14) and (4.17), we can now easily state our estimate on the problem (4.12).

Corollary 4.3. We consider the problem (4.12). We suppose that $F^{e} \in\left(V^{e}\right)^{\prime}, h_{0}^{e} \in H^{1}\left(\Gamma_{0}\right)$ and $h_{ \pm}^{e} \in H^{-1 / 2}\left(\Gamma_{ \pm}\right)$. Then we have

$$
\int_{\Omega}\left|\nabla_{e} \psi^{e}\right|^{2} d z \leq C\left(\left\|F^{e}\right\|_{\left(V^{e}\right)^{\prime}}+R_{3}\left(h_{0}^{e}, h_{+}^{e}, h_{-}^{e}\right)\right) .
$$

\subsection{Final results on problem (4.1)}

We now go back to problem (4.1) and derive estimates on the finite energy solution $\theta^{e}$. Combining the results of $\S 4.1, \S 4.2$, we obtain

Theorem 4.4. We suppose

$$
\left\{\begin{array}{l}
F^{e} \in L^{1}(0, T ; H), \theta_{0}^{e} \in H^{1}(\Omega), \frac{\partial \theta_{0}^{e}}{\partial \nu} \in H^{-1 / 2}\left(\Gamma_{ \pm}\right), \theta_{1}^{e} \in H \\
h_{0}^{e} \in C^{0}\left([0, T] ; H^{1}\left(\Gamma_{0}\right)\right) \cap H^{1}\left(0, T ; L^{2}\left(\Gamma_{0}\right)\right) \\
h_{ \pm}^{e} \in C^{0}\left([0, T] ; H^{-1 / 2}\left(\Gamma_{ \pm}\right)\right),\left(h_{ \pm}^{e}\right)^{\prime} \in\left(W_{ \pm}^{e}\right)^{\prime}
\end{array}\right.
$$

We further assume the compatibility condition (4.6). Then there exists a unique solution $\theta^{e}$ satisfying (4.1) with the following regularity:

$$
\left(\theta^{e},\left(\theta^{e}\right)^{\prime}\right) \in C^{0}\left([0, T] ; H^{1}(\Omega) \times H\right),\left(\theta^{e}\right)^{\prime \prime} \in L^{1}\left(0, T ;\left(V^{e}\right)^{\prime}\right)
$$

Further the following estimate holds:

$$
\begin{gathered}
\left\|\nabla_{e} \theta^{e}\right\|_{L^{\infty}(0, T ; H)}+\left\|\left(\theta^{e}\right)^{\prime}\right\|_{L^{\infty}(0, T ; H)} \leq \\
\leq C\left(\left\|F^{e}\right\|_{L^{1}(0, T ; H)}+R_{4}\left(\theta_{0}^{e}, \theta_{1}^{e}, h_{0}^{e}, h_{+}^{e}, h_{-}^{e}\right)\right),
\end{gathered}
$$




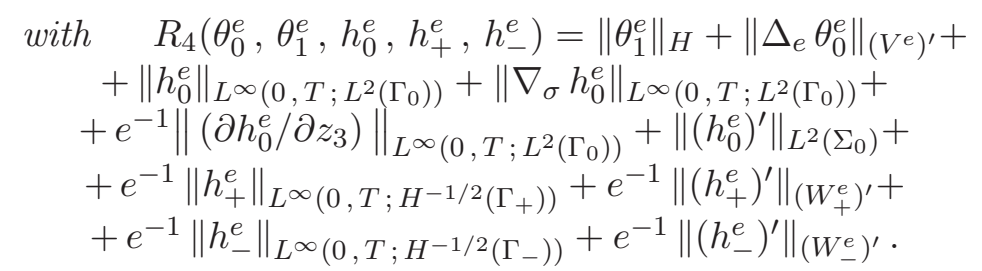

\section{Regularity in $z_{3}$-variable for solutions with fi- nite energy}

Let us consider the finite energy solution of system (4.1) whose existence and uniqueness have been proved in Theorem 4.4 . We are now interested in its regularity with respect to $z_{3}$-variable. To this end, we exploit the cylindrical structure of the domain. We introduce $\widetilde{\theta}^{e}=\partial \theta^{e} / \partial z_{3}$ and note that $\widetilde{\theta}^{e}$ satisfies

$$
\left\{\begin{array}{l}
\square_{e} \widetilde{\theta}^{e}=\widetilde{F}^{e} \text { in } Q, \\
\widetilde{\theta}^{e}=\widetilde{h}_{0}^{e} \text { on } \Sigma_{0} \text { and } e^{-1} \widetilde{\theta}^{e}=h_{ \pm}^{e} \nu_{3} \quad \text { on } \Sigma_{ \pm} \\
\widetilde{\theta}^{e}(0)=\widetilde{\theta}_{0}^{e} \text { and }\left(\widetilde{\theta}^{e}\right)^{\prime}(0)=\widetilde{\theta}_{1}^{e} \quad \text { in } \Omega
\end{array}\right.
$$

where we have posed

$$
\widetilde{F}^{e}=\left(\partial F^{e} / \partial z_{3}\right), \widetilde{h}_{0}^{e}=\left(\partial h_{0}^{e} / \partial z_{3}\right), \widetilde{\theta}_{0}^{e}=\left(\partial \theta_{0}^{e} / \partial z_{3}\right), \widetilde{\theta}_{1}^{e}=\left(\partial \theta_{1}^{e} / \partial z_{3}\right) .
$$

Let us note that (5.1) is an initial-boundary value problem with purely Dirichlet boundary conditions. A priori, we know that $\widetilde{\theta}^{e} \in$ $C^{0}([0, T] ; H)$ and $\left(\widetilde{\theta}^{e}\right)^{\prime} \in C^{0}\left([0, T] ;\left(V^{e}\right)^{\prime}\right)$, and so the problem (5.1) has to be understood in the weak sense. The goal of this section is to assume that the data in (5.1) are more regular in $z_{3}$ and prove that $\widetilde{\theta}^{e}$ is more regular in $z_{3}$. For this purpose, we need to derive estimates on the Dirichlet problem (5.1). 


\subsection{Problem with non-homogeneous Dirichlet boundary condition}

We begin by writing down the system which will be understood in a weak sense:

$$
\left\{\begin{array}{l}
\square_{e} \varphi^{e}=f^{e} \text { in } Q \\
\varphi^{e}=g_{0}^{e} \text { on } \Sigma_{0} \text { and } e^{-1} \varphi^{e}=g_{ \pm}^{e} \quad \text { on } \Sigma_{ \pm} \\
\varphi^{e}(0)=\varphi_{0}^{e} \text { and }\left(\varphi^{e}\right)^{\prime}(0)=\varphi_{1}^{e} \quad \text { in } \Omega
\end{array}\right.
$$

The meaning of this problem is analogous to that of (3.14) and is described in the following theorem, along with existence and uniqueness result. For the Dirichlet problem (5.2), the natural function space is $V^{e}=H_{0}^{1}(\Omega)$ with norm $\left\|\nabla_{e} v\right\|_{H^{3}}$. Its dual is denoted by $\left(V^{e}\right)^{\prime}$.

Theorem 5.1. We assume that the data in (5.2) satisfy

$$
\begin{aligned}
& f \in L^{1}\left(0, T ;\left(V^{e}\right)^{\prime}\right),\left(\varphi_{0}^{e}, \varphi_{1}^{e}\right) \in H \times\left(V^{e}\right)^{\prime} \\
& \left(g_{0}^{e}, g_{ \pm}^{e}\right) \in G L^{2}\left(\Sigma_{0}\right) \times L^{2}\left(\Sigma_{ \pm}\right)
\end{aligned}
$$

Then there exists a unique solution $\varphi^{e}$ such that

$$
\varphi^{e} \in C^{0}([0, T] ; H),\left(\varphi^{e}\right)^{\prime} \in C^{0}\left([0, T] ;\left(V^{e}\right)^{\prime}\right)
$$

and satisfying (5.2) in the following sense:

$$
\begin{aligned}
& L^{1}(0, T ; H)<G^{e}, \varphi^{e}>_{L^{\infty}(0, T ; H)}= \\
& ={ }_{L^{1}\left(0, T ;\left(V^{e}\right)^{\prime}\right)}<f^{e}, \chi^{e}>_{L^{\infty}\left(0, T ; V^{e}\right)}+ \\
& +\left(V^{e}\right)^{\prime}<\varphi_{1}^{e}, \chi^{e}(0)>_{V^{e}}- \\
& -{ }_{H}<\varphi_{0}^{e},\left(\chi^{e}\right)^{\prime}(0)>_{H}-{ }_{L^{2}\left(\Sigma_{0}\right)}<\left(\partial \chi^{e} / \partial \nu\right), g_{0}^{e}>_{L^{2}\left(\Sigma_{0}\right)}- \\
& -e_{L^{2}\left(\Sigma_{+}\right)}^{-1}<\left(\partial \chi^{e} / \partial \nu\right), g_{+}^{e}>_{L^{2}\left(\Sigma_{+}\right)}- \\
& -e_{L^{2}\left(\Sigma_{-}\right)}^{-1}<\left(\partial \chi^{e} / \partial \nu\right), g_{-}^{e}>_{L^{2}\left(\Sigma_{-}\right)},
\end{aligned}
$$

for all $G^{e} \in L^{1}(0, T ; H)$ and for all finite energy solutions $\chi^{e}$ of the following backward homogeneous Dirichlet initial-boundary value problem:

$$
\left\{\begin{array}{l}
\square_{e} \chi^{e}=G^{e} \quad \text { in } Q \\
\chi^{e}=0 \text { on } \Sigma_{0} \cup \Sigma_{ \pm}, \\
\chi^{e}(T)=\left(\chi^{e}\right)^{\prime}(T)=0 \quad \text { in } \Omega
\end{array}\right.
$$


Moreover, we have the following estimates:

$$
\left\{\begin{array}{c}
\left\|\varphi^{e}\right\|_{L^{\infty}(0, T ; H)}+\left\|\left(\varphi^{e}\right)^{\prime}\right\|_{L^{\infty}\left(0, T ;\left(V^{e}\right)^{\prime}\right)} \leq \\
\leq C R_{5}\left(f^{e}, \varphi_{0}^{e}, \varphi_{1}^{e}, g_{0}^{e}, g_{+}^{e}, g_{-}^{e}\right) \\
\text { with } R_{5}\left(f^{e}, \varphi_{0}^{e}, \varphi_{1}^{e}, g_{0}^{e}, g_{+}^{e}, g_{-}^{e}\right) \equiv \\
\equiv\left\|f^{e}\right\|_{L^{1}\left(0, T ;\left(V^{e}\right)^{\prime}\right)}+ \\
+\left\|\varphi_{0}^{e}\right\|_{H}+\left\|\varphi_{1}^{e}\right\|_{\left(V^{e}\right)^{\prime}}+\left\|g_{0}^{e}\right\|_{L^{2}\left(\Sigma_{0}\right)}+ \\
+\left\|g_{+}^{e}\right\|_{L^{2}\left(\Sigma_{+}\right)}+\left\|g_{-}^{e}\right\|_{L^{2}\left(\Sigma_{-}\right)}
\end{array}\right.
$$

Proof. The above result has been proved by Lions [6] in the case where there is no parameter $e$ in the problem. The effect of this parameter can be analyzed as indicated in Section 3, in the case of mixed boundary conditions. More precisely, for the proof of (5.7) we consider solutions $\chi^{e}$ of (5.6) with $G^{e}=\left(G_{1}^{e}\right)^{\prime}$ where $G_{1}^{e} \in L^{1}\left(0, T ; V^{e}\right)$ and establish

$$
\begin{aligned}
\left\|\nabla_{e} \chi^{e}\right\|_{L^{\infty}(0, T ; H)}+ & \left\|\left(\chi^{e}\right)^{\prime}\right\|_{L^{\infty}(0, T ; H)}+\left\|\left(\partial \chi^{e} / \partial \nu\right)\right\|_{L^{2}\left(\Sigma_{0}\right)}+ \\
& +e^{-1}\left\|\left(\partial \chi^{e} / \partial \nu\right)\right\|_{L^{2}\left(\Sigma_{+} \cup \Sigma_{-}\right)} \leq \\
& \leq C\left\|\nabla_{e} G_{1}^{e}\right\|_{L^{1}(0, T ; H)} .
\end{aligned}
$$

This requires the introduction of another type of multipliers $m_{k}^{ \pm} \in$ $W^{1, \infty}(\Omega), k=1,2,3$ different from (3.13) and they satisfy:

$$
\left\{\begin{array}{l}
m_{1}^{ \pm}, m_{2}^{ \pm} \quad \text { independent of } z_{3}, \quad m_{\alpha}^{ \pm}=0 \quad \text { on } \Gamma_{0}, \\
m_{3}^{ \pm}=\nu_{3} \text { on } \Gamma_{ \pm} \text {and } m_{3}^{+}=0 \text { on } \Gamma_{-}, m_{3}^{-}=0 \text { on } \Gamma_{+} .
\end{array}\right.
$$

Let us now turn our attention towards solutions with finite energy for non-homogeneous Dirichlet problems. Thus, we consider

$$
\left\{\begin{array}{l}
\square_{e} \chi^{e}=G^{e} \text { in } Q, \\
\chi^{e}=\ell_{0}^{e} \text { on } \Sigma_{0} \text { and } e^{-1} \chi^{e}=\ell_{ \pm}^{e} \text { on } \Sigma_{ \pm} \\
\chi^{e}(0)=\chi_{0}^{e} \text { and }\left(\chi^{e}\right)^{\prime}(0)=\chi_{1}^{e} \text { in } \Omega
\end{array}\right.
$$

Treatment of this problem is similar to that of (4.1) and one can indeed prove

Theorem 5.2. We make the following regularity and compatibility assumptions

$$
\left\{\begin{array}{l}
G^{e} \in L^{1}(0, T ; H), \chi_{0}^{e} \in H^{1}(\Omega), \chi_{1}^{e} \in H \\
\ell_{0}^{e} \in C^{0}\left([0, T] ; H^{1}\left(\Gamma_{0}\right)\right) \cap H^{1}\left(0, T ; L^{2}\left(\Gamma_{0}\right)\right) \\
\ell_{ \pm}^{e} \in C^{0}\left([0, T] ; H^{1}\left(\Gamma_{ \pm}\right)\right) \cap H^{1}\left(0, T ; L^{2}\left(\Gamma_{ \pm}\right)\right)
\end{array}\right.
$$




$$
\left.\ell_{0}^{e}\right|_{t=0}=\chi_{0}^{e} \quad \text { on } \Gamma_{0} \quad \text { and }\left.\quad \ell_{ \pm}^{e}\right|_{t=0}=e^{-1} \chi_{0}^{e} \quad \text { on } \Gamma_{ \pm} .
$$

Then, there exists a unique solution $\chi^{e}$ to (5.10) with the following regularity:

$$
\chi^{e} \in C^{0}\left([0, T] ; H^{1}(\Omega)\right),\left(\chi^{e}\right)^{\prime} \in C^{0}([0, T] ; H) .
$$

Moreover, we have the estimate

$$
\begin{aligned}
& \left\|\nabla_{e} \chi^{e}\right\|_{L^{\infty}(0, T ; H)^{3}}+\left\|\left(\chi^{e}\right)^{\prime}\right\|_{L^{\infty}(0, T ; H)} \leq \\
& \leq C\left(\left\|G^{e}\right\|_{L^{1}(0, T ; H)}+R_{6}\left(\chi_{0}^{e}, \chi_{1}^{e}, \ell_{0}^{e}, \ell_{+}^{e}, \ell_{-}^{e}\right)\right. \\
& \text { with } R_{6}\left(\chi_{0}^{e}, \chi_{1}^{e}, \ell_{0}^{e}, \ell_{+}^{e}, \ell_{-}^{e}\right) \equiv\left\|\chi_{0}^{e}\right\|_{H}+ \\
& +\left\|\Delta_{e} \chi_{0}^{e}\right\|_{(V e)^{\prime}}+\left\|\chi_{1}^{e}\right\|_{H}+ \\
& +\left\|\ell_{0}^{e}\right\|_{L^{\infty}\left(0, T ; L^{2}\left(\Gamma_{0}\right)\right)}+\left\|\nabla_{\sigma} \ell_{0}^{e}\right\|_{L^{\infty}\left(0, T ; L^{2}\left(\Gamma_{0}\right)\right)}+ \\
& +e^{-1}\left\|\partial \ell_{0}^{e} / \partial z_{3}\right\|_{L^{\infty}\left(0, T ; L^{2}\left(\Gamma_{0}\right)\right)}+ \\
& +\left\|\left(\ell_{0}^{e}\right)^{\prime}\right\|_{L^{2}\left(\Sigma_{0}\right)}+\left\|\ell_{+}^{e}\right\|_{H^{1}\left(0, T ; L^{2}\left(\Gamma_{+}\right)\right)}+\| \ell_{+}^{e} \\
& +\|\|_{L^{\infty}\left(0, T ; H^{1}\left(\Gamma_{+}\right)\right)}+\left\|\ell_{-}^{e}\right\|_{H^{1}\left(0, T ; L^{2}\left(\Gamma_{-}\right)\right)}+\left\|\ell_{-}^{e}\right\|_{L^{\infty}\left(0, T ; H^{1}\left(\Gamma_{-}\right)\right)} .
\end{aligned}
$$

\subsection{Regularity in $z_{3}$-variable}

We establish the regularity of finite energy solutions $\theta^{e}$ of (4.1) with respect to $z_{3}$-variable under suitable additional assumptions on the data. We impose conditions on $\widetilde{F}^{e}, \widetilde{h}_{0}^{e}, \widetilde{h}_{ \pm}^{e}, \widetilde{\theta}_{0}^{e}, \widetilde{\theta}_{1}^{e}$ which will imply existence of a finite energy solution to (5.1) (via Theorem 5.2). Let us record these results below.

Theorem 5.3. With reference to problem (4.1), let us make the hypothesis that the data satisfy (4.6), (4.25) and in addition that

$$
\left\{\begin{array}{l}
\frac{\partial F^{e}}{\partial z_{3}} \in L^{1}(0, T ; H), \frac{\partial \theta_{0}^{e}}{\partial z_{3}} \in H^{1}(\Omega), \frac{\partial \theta_{1}^{e}}{\partial z_{3}} \in H, \\
\frac{\partial h_{0}^{e}}{\partial z_{3}} \in C^{0}\left([0, T] ; H^{1}\left(\Gamma_{0}\right)\right) \cap H^{1}\left(0, T ; L^{2}\left(\Gamma_{0}\right)\right), \\
h_{ \pm}^{e} \in C^{0}\left([0, T] ; H^{1}\left(\Gamma_{ \pm}\right)\right) \cap H^{1}\left(0, T ; L^{2}\left(\Gamma_{ \pm}\right)\right) .
\end{array}\right.
$$

Then the solution $\theta^{e}$ has the additional regularity (apart from the one announced in Theorem 4.4):

$$
\frac{\partial \theta^{e}}{\partial z_{3}} \in C^{0}\left([0, T] ; H^{1}(\Omega)\right), \frac{\partial\left(\theta^{e}\right)^{\prime}}{\partial z_{3}} \in C^{0}\left([0, T] ; L^{2}(\Omega)\right) .
$$


Moreover, we have the estimate

$$
\begin{aligned}
& \left\|\nabla_{e}\left(\partial \theta^{e} / \partial z_{3}\right)\right\|_{L^{\infty}(0, T ; H)^{3}}+\left\|\left(\partial\left(\theta^{e}\right)^{\prime} / \partial z_{3}\right)\right\|_{L^{\infty}(0, T ; H)} \leq \\
& \left.\leq C\left(\left\|\frac{\partial F^{e}}{\partial z_{3}}\right\|_{L^{1}(0, T ; H)}+R_{6} \frac{\partial \theta_{0}^{e}}{\partial z_{3}}, \frac{\partial \theta_{1}^{e}}{\partial z_{3}}, \frac{\partial h_{0}^{e}}{\partial z_{3}}, h_{+}^{e}, h_{-}^{e}\right)\right) .
\end{aligned}
$$

Using classical trace results, we derive, from Theorem 5.3, the following:

Corollary 5.4. Under the assumptions of Theorem 5.3, we have

$$
\left.\theta^{e}\right|_{\Sigma_{ \pm}} \in C^{0}\left([0, T] ; H^{1}\left(\Gamma_{ \pm}\right)\right),\left.\left(\theta^{e}\right)^{\prime}\right|_{\Sigma_{ \pm}} \in C^{0}\left([0, T] ; L^{2}\left(\Gamma_{ \pm}\right)\right) .
$$

Further, the estimates below hold

$$
\begin{aligned}
& \left\|\theta^{e}\right\|_{L^{\infty}\left(0, T ; H^{1}\left(\Gamma_{ \pm}\right)\right)}+\left\|\left(\theta^{e}\right)^{\prime}\right\|_{L^{\infty}\left(0, T ; L^{2}\left(\Gamma_{ \pm}\right)\right)} \leq \\
& \leq C\left\{\left\|F^{e}\right\|_{L^{1}(0, T ; H)}+\left\|\left(\partial F^{e} / \partial z_{3}\right)\right\|_{L^{1}(0, T ; H)}+\right. \\
& +R_{4}\left(\theta_{0}^{e}, \theta_{1}^{e}, h_{0}^{e}, h_{+}^{e}, h_{-}^{e}\right)+ \\
& \left.+R_{6}\left(\partial \theta_{0}^{e} / \partial z_{3}, \partial \theta_{1}^{e} / \partial z_{3}, \partial h_{0}^{e} / \partial z_{3}, h_{+}^{e}, h_{-}^{e}\right)\right\} .
\end{aligned}
$$

\section{Regular solutions of (4.1)}

We now establish a result which gives regularity of finite energy solutions to (4.1) by imposing additional hypotheses on the data. The index $s$ appearing in the next theorem is due to the fact that our domain $\Omega$ is cyclindrical and we have mixed boundary conditions. This index $s$ is the same as the one which occurs in the regularity of stationary mixed boundary value problems (see Grisvard [2]).

Theorem 6.1. With reference to problem (4.1), we suppose

$$
\left\{\begin{array}{l}
F^{e} \in L^{1}\left(0, T ; V^{e}\right), \theta_{0}^{e} \in H^{2}(\Omega), \theta_{1}^{e} \in H^{1}(\Omega) \\
h_{0}^{e} \in C^{0}\left([0, T] ; H^{3 / 2}\left(\Gamma_{0}\right)\right),\left(h_{0}^{e}\right)^{\prime} \in C^{0}\left([0, T] ; H^{1 / 2}\left(\Gamma_{0}\right)\right) \\
\left(h_{0}^{e}\right)^{\prime \prime} \in L^{1}\left(0, T ; H^{1 / 2}\left(\Gamma_{0}\right)\right), h_{ \pm}^{e} \in C^{0}\left([0, T] ; H^{1 / 2}\left(\Gamma_{ \pm}\right)\right) \\
\left(h_{ \pm}^{e}\right)^{\prime} \in C^{0}\left([0, T] ; H^{-1 / 2}\left(\Gamma_{ \pm}\right)\right),\left(h_{ \pm}^{e}\right)^{\prime \prime} \in L^{1}\left(0, T ; H^{-1 / 2}\left(\Gamma_{ \pm}\right)\right)
\end{array}\right.
$$

Further, we assume that the following compatibility conditions are satisfied

$$
\begin{aligned}
& \theta_{0}^{e}=\left.h_{0}^{e}\right|_{t=0} \quad \text { on } \Gamma_{0}, \theta_{1}^{e}=\left.\left(h_{0}^{e}\right)^{\prime}\right|_{t=0} \quad \text { on } \Gamma_{0} \\
& e^{-1} \frac{\partial \theta_{0}^{e}}{\partial \nu}=\left.h_{ \pm}^{e}\right|_{t=0} \text { on } \Gamma_{ \pm} .
\end{aligned}
$$


Then, there exists $s$, with $3 / 2<s<2$, such that the solution $\theta^{e}$ of (4.1) has the regularity

$$
\theta^{e} \in C^{0}\left([0, T] ; H^{s}(\Omega)\right), \quad\left(\theta^{e}\right)^{\prime} \in C^{0}\left([0, T] ; H^{1}(\Omega)\right) .
$$

Proof. We introduce the stationary problem with non-homogeneous boundary data parametrized by $t$ :

$$
\left\{\begin{array}{l}
-\Delta_{e} H^{e}(t)=0 \quad \text { in } \Omega, \\
H^{e}(t)=h_{0}^{e}(t) \quad \text { on } \Gamma_{0} \quad \text { and } \quad e^{-1} \frac{\partial H^{e}}{\partial \nu}(t)=h_{ \pm}^{e}(t) \quad \text { on } \Gamma_{ \pm}
\end{array}\right.
$$

Applying the trace theorem from Grisvard [1] p. 230, for each fixed $t$, we can lift the boundary data $h_{0}^{e}(t), h_{ \pm}^{e}(t)$ to a function in $H^{s}(\Omega)$, for some $s$ such that $3 / 2<s<2$, without compatibility condition. This enables us to transform the problem (6.4) into a problem with homogeneous boundary data and a right-hand side belonging to $H^{s-2}(\Omega)$. It now follows, from Grisvard [2], that there exists $s$, with $3 / 2<s<2$ such that

$$
H^{e} \in C^{0}\left([0, T] ; H^{s}(\Omega)\right) .
$$

Thanks to our hypotheses on $\left(h_{0}^{e}\right)^{\prime},\left(h_{0}^{e}\right)^{\prime \prime},\left(h_{ \pm}^{e}\right)^{\prime}$ and $\left(h_{ \pm}^{e}\right)^{\prime \prime}$, we deduce that

$$
\left(H^{e}\right)^{\prime} \in C^{0}\left([0, T] ; H^{1}(\Omega)\right), \quad\left(H^{e}\right)^{\prime \prime} \in C^{0}\left([0, T] ; H^{1}(\Omega)\right) .
$$

The next step is to consider $\widetilde{\theta}^{e}(t)=\theta^{e}(t)-H^{e}(t)$. Then $\widetilde{\theta}^{e}$ satisfies

$$
\left\{\begin{array}{l}
\square^{\tilde{\theta}^{e}}=F^{e}-\left(H^{e}\right)^{\prime \prime} \text { in } Q \\
\widetilde{\theta}^{e}=0 \text { on } \Sigma_{0} \text { and } e^{-1}\left(\partial \widetilde{\theta}^{e} / \partial \nu\right)=0 \quad \text { on } \Sigma_{ \pm}, \\
\widetilde{\theta}^{e}(0)=\theta_{0}^{e}-H^{e}(0) \text { and }\left(\widetilde{\theta}^{e}\right)^{\prime}(0)=\theta_{1}^{e}-\left(H^{e}\right)^{\prime}(0) \quad \text { in } \Omega
\end{array}\right.
$$

From the conditions (6.2), it follows that $\theta_{0}^{e}-H^{e}(0) \in H^{s}(\Omega) \cap V^{e}$, $\theta_{1}^{e}-\left(H^{e}\right)^{\prime}(0) \in V^{e}$ and $(\partial / \partial \nu)\left(\theta_{0}^{e}-H^{e}(0)\right)=0$ on $\Gamma_{ \pm}$. This enables us to apply once more the regularity results of Grisvard [2] to $\widetilde{\theta}^{e}$ and this gives the result (6.3) on $\theta^{e}$. 


\section{Direct inequality for finite energy solutions with non homogeneous mixed boundary con- ditions}

We begin by remarking that the result in the homogeneous case has been proved in Saint Jean Paulin \& Vanninathan [8], and we follow the same method to treat the present non-homogeneous case. The main result of this section (referred to as Direct Inequality in the literature) shows that the normal derivative $\partial \theta^{e} / \partial \nu$ of the solution of (4.1) is in $L^{2}\left(\Sigma_{0}\right)$. It is to be stressed that this property is automatic for homogeneous solutions with finite energy, whereas, in the non-homogeneous case it holds only under additional hypotheses on data. This will be made clear by the identity established in the result that follows.

Theorem 7.1. We consider the problem (4.1) and assume that the data satisfy (4.6), (4.25) and (5.15). Then, for all multipliers $m_{k} \in$ $W^{1, \infty}(\Omega), k=1,2,3$, the solution $\theta^{e}$ satisfies the following identity:

$$
\begin{aligned}
& \frac{1}{2} \int_{\Sigma_{0}}\left(m_{\alpha} \nu_{\alpha}\right)\left(\frac{\partial \theta^{e}}{\partial \nu}\right)^{2} d \sigma d t+ \\
& +\frac{1}{2} \int_{\Sigma_{+} \cup \Sigma_{-}}\left(m_{3} \nu_{3}\right)\left\{\left(\frac{\partial \theta^{e}}{\partial t}\right)^{2}-\frac{\partial \theta^{e}}{\partial z_{\alpha}} \frac{\partial \theta^{e}}{\partial z_{\alpha}}\right\} d \sigma d t= \\
& =\left[\int_{\Omega} \frac{\partial \theta^{e}}{\partial t} m_{k} \frac{\partial \theta^{e}}{\partial z_{k}} d z\right]_{0}^{T}+ \\
& +\frac{1}{2} \int_{Q} \frac{\partial m_{k}}{\partial z_{k}}\left\{\left(\frac{\partial \theta^{e}}{\partial t}\right)^{2}-\frac{\partial \theta^{e}}{\partial z_{\alpha}} \frac{\partial \theta^{e}}{\partial z_{\alpha}}-e^{-2}\left(\frac{\partial \theta^{e}}{\partial z_{3}}\right)^{2}\right\} d z d t+ \\
& +\int_{Q} \frac{\partial m_{k}}{\partial z_{\alpha}} \frac{\partial \theta^{e}}{\partial z_{\alpha}} \frac{\partial \theta^{e}}{\partial z_{k}} d z d t+e^{-2} \int_{Q} \frac{\partial m_{k}}{\partial z_{3}} \frac{\partial \theta^{e}}{\partial z_{3}} \frac{\partial \theta^{e}}{\partial z_{k}} d z d t- \\
& -\int_{Q} F^{e} m_{k} \frac{\partial \theta^{e}}{\partial z_{k}} d z d t- \\
& -\frac{1}{2} \int_{\Sigma_{0}}\left(m_{\alpha} \nu_{\alpha}\right)\left\{\left(\frac{\partial h_{0}^{e}}{\partial t}\right)^{2}-\sigma_{\beta} h_{0}^{e} \sigma_{\beta} h_{0}^{e}-e^{-2}\left(\frac{\partial h_{0}^{e}}{\partial z_{3}}\right)^{2}\right\} d \sigma d t- \\
& -\int_{\Sigma_{0}} \frac{\partial \theta^{e}}{\partial \nu}\left\{m_{\alpha} \sigma_{\alpha} h_{0}^{e}+m_{3} \frac{\partial h_{0}^{e}}{\partial z_{3}}\right\} d \sigma d t- \\
& -\frac{1}{2} \int_{\Sigma_{+}}\left(m_{3} \nu_{3}\right)\left(h_{+}^{e}\right)^{2} d \sigma d t-\frac{1}{2} \int_{\Sigma_{-}}\left(m_{3} \nu_{3}\right)\left(h_{-}^{e}\right)^{2} d \sigma d t- \\
& -e^{-1} \int_{\Sigma_{+}} h_{+}^{e} m_{\alpha} \frac{\partial \theta^{e}}{\partial z_{\alpha}} d \sigma d t-e^{-1} \int_{\Sigma_{-}} h_{-}^{e} m_{\alpha} \frac{\partial \theta^{e}}{\partial z_{\alpha}} d \sigma d t .
\end{aligned}
$$


Proof. The proof of the above identity is done in two steps.

Step 1. We establish the identity (7.1) for solution $\theta^{e}$ of (4.1) with data satisfying (4.6), (4.25) and (5.15) and having the additional properties assumed in Theorem 6.1.

Step 2. By density arguments, we conclude that (7.1) remains valid for solutions $\theta^{e}$ when data satisfy (4.6), (4.25) and (5.15).

Proof of Step 1. Following Lions [6], we multiply the equation in (4.1) by $m_{k} \partial \theta^{e} / \partial z_{k}$ and integrate by parts (which is allowed because the solution is smooth by Theorem 6.1). Now, we have

$$
\begin{aligned}
& \int_{Q} F^{e} m_{k} \frac{\partial \theta^{e}}{\partial z_{k}} d z d t=\left[\int_{\Omega}\left(\theta^{e}\right)^{\prime} m_{k} \frac{\partial \theta^{e}}{\partial z_{k}} d z\right]_{0}^{T}+ \\
& +\frac{1}{2} \int_{Q} \frac{\partial m_{k}}{\partial z_{k}}\left|\left(\theta^{e}\right)^{\prime}\right|^{2} d z d t-\frac{1}{2} \int_{\Sigma_{0}}\left(m_{\alpha} \nu_{\alpha}\right)\left|\left(h_{0}^{e}\right)^{\prime}\right|^{2} d \sigma d t- \\
& -\frac{1}{2} \int_{\Sigma_{+} \cup \Sigma_{-}}\left(m_{3} \nu_{3}\right)\left|\left(\theta^{e}\right)^{\prime}\right|^{2} d \sigma d t-\int_{Q}\left(\Delta_{e} \theta^{e}\right) m_{k} \frac{\partial \theta^{e}}{\partial z_{k}} d z d t
\end{aligned}
$$

We will now do the computation of the last integral separately.

$$
\begin{aligned}
& \int_{Q}\left(\Delta_{e} \theta^{e}\right) m_{k} \frac{\partial \theta^{e}}{\partial z_{k}} d z d t=- \\
& -\int_{Q} \frac{\partial \theta^{e}}{\partial z_{\alpha}} \frac{\partial m_{k}}{\partial z_{\alpha}} \frac{\partial \theta^{e}}{\partial z_{k}} d z d t-e^{-2} \int_{Q} \frac{\partial \theta^{e}}{\partial z_{3}} \frac{\partial m_{k}}{\partial z_{3}} \frac{\partial \theta^{e}}{\partial z_{k}} d z d t+ \\
& +\frac{1}{2} \int_{Q} \frac{\partial m_{k}}{\partial z_{k}}\left\{\frac{\partial \theta^{e}}{\partial z_{\alpha}} \frac{\partial \theta^{e}}{\partial z_{\alpha}}+e^{-2}\left(\frac{\partial \theta^{e}}{\partial z_{3}}\right)^{2}\right\} d z d t+ \\
& +\int_{\Sigma_{0}} \frac{\partial \theta^{e}}{\partial z_{\alpha}} \nu_{\alpha} m_{k} \frac{\partial \theta^{e}}{\partial z_{k}} d \sigma d t+ \\
& +e^{-2} \int_{\Sigma_{+} \cup \Sigma_{-}} \frac{\partial \theta^{e}}{\partial z_{3}} \nu_{3} m_{k} \frac{\partial \theta^{e}}{\partial z_{k}} d \sigma d t- \\
& -\frac{1}{2} \int_{\Sigma_{0}}\left(m_{\beta} \nu_{\beta}\right)\left\{\frac{\partial \theta^{e}}{\partial z_{\alpha}} \frac{\partial \theta^{e}}{\partial z_{\alpha}}+e^{-2}\left(\frac{\partial \theta^{e}}{\partial z_{3}}\right)^{2}\right\} d z d t- \\
& -\frac{1}{2} \int_{\Sigma_{+} \cup \Sigma_{-}}\left(m_{3} \nu_{3}\right)\left\{\frac{\partial \theta^{e}}{\partial z_{\alpha}} \frac{\partial \theta^{e}}{\partial z_{\alpha}}+e^{-2}\left(\frac{\partial \theta^{e}}{\partial z_{3}}\right)^{2}\right\} d \sigma d t
\end{aligned}
$$

We use the following relations

$$
\left\{\begin{array}{l}
e^{-1}\left(\partial \theta^{e} / \partial z_{3}\right) \nu_{3}=e^{-1}\left(\partial \theta^{e} / \partial \nu\right)=h_{ \pm}^{e} \text { on } \Sigma_{ \pm} \\
\frac{\partial \theta^{e}}{\partial z_{\alpha}}=\nu_{\alpha} \frac{\partial \theta^{e}}{\partial \nu}+\sigma_{\alpha} h_{0}^{e} \text { on } \Sigma_{0} \text { and } \nu_{\alpha} \sigma_{\alpha} h_{0}^{e}=0 \text { on } \Sigma_{0}
\end{array}\right.
$$


Use of (7.4) in (7.3) yields (7.1) and this completes Step 1.

Proof of Step 2. On one hand, it is obvious that data satisfying (4.6), (4.25) and (5.15) can be approximated by those satisfying additional hypotheses of Theorem 6.1. On the other hand, each term of the identity (7.1) established in Step 1 remains continuous with respect to data satisfying (4.6), (4.25) and (5.15). This ends the proof.

The last two terms in (7.1) are especially troublesome. To bound them, we need hypothesis (5.15), Corollary 5.4 and hence the regularity result w.r.t $z_{3}$ in cylindrical domains. All other terms can be estimated with the hypotheses under which we obtain energy estimates (cf. Theorem 4.4) and these are available for general domains.

Corollary 7.2. Under the hypotheses of Theorem 7.1, we have $\partial \theta^{e} / \partial \nu \in$ $L^{2}\left(\Sigma_{0}\right)$ and we have the estimate

$$
\begin{aligned}
& \left\|\left(\partial \theta^{e} / \partial \nu\right)\right\|_{L^{2}\left(\Sigma_{0}\right)} \leq C\left\{\left\|F^{e}\right\|_{L^{1}(0, T ; H)}+R_{4}\left(\theta_{0}^{e}, \theta_{1}^{e}, h_{0}^{e}, h_{+}^{e}, h_{-}^{e}\right)+\right. \\
& \left.+R_{6}\left(\partial \theta_{0}^{e} / \partial z_{3}, \partial \theta_{1}^{e} / \partial z_{3}, \partial h_{0}^{e} / \partial z_{3}, h_{+}^{e}, h_{-}^{e}\right)\right\}
\end{aligned}
$$

where $R_{4}$ and $R_{6}$ are defined by (4.28) and (5.14) respectively.

Proof. We decompose the solution into two parts: $\theta^{e}=\bar{\theta}^{e}+\left(\theta^{e}-\bar{\theta}^{e}\right)$, where $\bar{\theta}^{e}$ and $\left(\theta^{e}-\bar{\theta}^{e}\right)$ are solutions of finite energy of (4.2) and (4.3) respectively.

The estimate of $(\partial / \partial \nu)\left(\theta^{e}-\bar{\theta}^{e}\right)$ in $L^{2}\left(\Sigma_{0}\right)$ is already announced in (3.7):

$$
\left\|(\partial / \partial \nu)\left(\theta^{e}-\bar{\theta}^{e}\right)\right\|_{L^{2}\left(\Sigma_{0}\right)} \leq C\left\|F^{e}\right\|_{L^{1}(0, T ; H)} .
$$

To estimate $\partial \theta^{e} / \partial \nu$ in $L^{2}\left(\Sigma_{0}\right)$, we use the identity (7.1), Theorem 4.4 and Corollary 5.4.

Remark 7.3. Let $\varphi^{e}$ be a solution with finite energy of problem (4.1) with $F^{e}=0, h_{0}^{e}=h_{ \pm}^{e}=0$ and with initial conditions $\left(\varphi_{0}^{e}, \varphi_{1}^{e}\right)$. Then, 
performing calculations analogous to (7.1), we obtain

$$
\begin{aligned}
& \int_{\Sigma_{0}}\left(m_{\beta} \nu_{\beta}\right) \frac{\partial \varphi^{e}}{\partial \nu} \frac{\partial \theta^{e}}{\partial \nu} d \sigma d t+ \\
& +\int_{\Sigma_{+} \cup \Sigma_{-}}\left(m_{3} \nu_{3}\right)\left\{\frac{\partial \varphi^{e}}{\partial t} \frac{\partial \theta^{e}}{\partial t}-\frac{\partial \varphi^{e}}{\partial z_{\alpha}} \frac{\partial \theta^{e}}{\partial z_{\alpha}}\right\} d \sigma d t= \\
& =-\int_{Q} F^{e} m_{k} \frac{\partial \varphi^{e}}{\partial z_{k}} d z d t+ \\
& +\left[\int_{\Omega}\left\{\frac{\partial \theta^{e}}{\partial t} m_{k} \frac{\partial \varphi^{e}}{\partial z_{k}}+\frac{\partial \varphi^{e}}{\partial t} m_{k} \frac{\partial \theta^{e}}{\partial z_{k}}\right\} d z\right]_{0}^{T}+ \\
& +\int_{Q} \frac{\partial m_{k}}{\partial z_{k}}\left\{\frac{\partial \varphi^{e}}{\partial t} \frac{\partial \theta^{e}}{\partial t}-\nabla_{e} \varphi^{e} \cdot \nabla_{e} \theta^{e}\right\} d z d t+ \\
& +\int_{Q}^{Q} \frac{\partial m_{k}}{\partial z_{\alpha}}\left\{\frac{\partial \varphi^{e}}{\partial z_{k}} \frac{\partial \theta^{e}}{\partial z_{\alpha}}+\frac{\partial \theta^{e}}{\partial z_{k}} \frac{\partial \varphi^{e}}{\partial z_{\alpha}}\right\} d z d t+ \\
& +e^{-2} \int_{Q} \frac{\partial m_{k}}{\partial z_{3}}\left\{\frac{\partial \varphi^{e}}{\partial z_{k}} \frac{\partial \theta^{e}}{\partial z_{3}}+\frac{\partial \varphi^{e}}{\partial z_{3}} \frac{\partial \theta^{e}}{\partial z_{k}}\right\} d z d t .
\end{aligned}
$$

By making the choice of multipliers (5.9), we see that

$$
\begin{aligned}
& \left|\int_{\Sigma_{ \pm}}\left(\left(\varphi^{e}\right)^{\prime}\left(\theta^{e}\right)^{\prime}-\left(\partial \varphi^{e} / \partial z_{\alpha}\right)\left(\partial \theta^{e} / \partial z_{\alpha}\right)\right) d \sigma d t\right| \leq \\
& \leq C E^{e}\left(\varphi^{e} ; 0\right)^{1 / 2}\left\{\left\|F^{e}\right\|_{L^{1}(0, T ; H)}+\right. \\
& \left.+\sup _{0 \leq t \leq T} E^{e}\left(\theta^{e} ; t\right)^{1 / 2}+\left\|\left(\partial h_{0}^{e} / \partial z_{3}\right)\right\|_{L^{2}\left(\Sigma_{0}\right)}\right\} .
\end{aligned}
$$

Thus we get an estimate merely under the hypotheses of Theorem 4.4. On the other hand, to estimate $\left|\int_{\Sigma_{0}}\left(\partial \varphi^{e} / \partial \nu\right)\left(\partial \theta^{e} / \partial \nu\right) d \sigma d t\right|$, we make the choice of multipliers

$$
m_{\alpha} \text { independent of } z_{3} \quad m_{\alpha}=\nu_{\alpha} \text { on } \gamma, \quad m_{3} \equiv 0 \text {, }
$$

and require additional hypothesis (5.15). Thus, somewhat surprisingly, $\theta^{e}$ on $\Sigma_{ \pm}$behaves better than expected.

Remark 7.4. In Theorem 7.1, if we replace the assumption (5.15) by

$$
h_{ \pm}^{e} \in C^{0}\left([0, T] ; H_{0}^{1}\left(\Gamma_{ \pm}\right)\right) \quad \text { and } \quad m_{\alpha} \in W^{2, \infty}(\Omega) \text {, }
$$


then relation (7.1) remains valid with the last two terms replaced by

$$
+e^{-1} \int_{\Sigma_{+}} \frac{\partial\left(h_{+}^{e} m_{\alpha}\right)}{\partial z_{\alpha}} \theta^{e} d \sigma d t+e^{-1} \int_{\Sigma_{-}} \frac{\partial\left(h_{-}^{e} m_{\alpha}\right)}{\partial z_{\alpha}} \theta^{e} d \sigma d t
$$

The proof uses usual density arguments. This trick shows a different way of managing the troublesome terms in (7.1) without using regularity w.r.t $z_{3}$.

\section{Construction of boundary sentinels}

We consider the original problem of sentinels posed in Section 2. We start by stating a result which ensures existence of a unique finite energy solution to the problem (2.2). This result is an easy consequence of Theorem 4.4, Corollary 5.4 and Corollary 7.2 and the fact that $F^{e} \equiv 0$.

Theorem 8.1. With regard to problem (2.2), we suppose that

$$
\begin{aligned}
& \left(y_{0}^{e}, \widehat{y}_{0}^{e}, \frac{\partial y_{0}^{e}}{\partial z_{3}}, \frac{\partial \widehat{y}_{0}^{e}}{\partial z_{3}}\right) \in\left(H^{1}(\Omega)\right)^{4},\left(\frac{\partial y_{0}^{e}}{\partial \nu}, \frac{\partial \widehat{y}_{0}^{e}}{\partial \nu}\right) \in\left(H^{-1 / 2}\left(\Gamma_{ \pm}\right)\right)^{2}, \\
& \left(y_{1}^{e}, \widehat{y}_{1}^{e}, \frac{\partial y_{1}^{1}}{\partial z_{3}}, \frac{\partial y}{\partial y_{3}}\right) \in H^{4}, \\
& \left(h_{0}^{e}, \widehat{h}_{0}^{e}, \frac{\partial h_{0}^{e}}{\partial z_{3}}, \frac{\partial \widehat{h}_{0}^{e}}{\partial z_{3}}\right) \in\left[C^{0}\left([0, T] ; H^{1}\left(\Gamma_{0}\right)\right) \cap H^{1}\left(0, T ; L^{2}\left(\Gamma_{0}\right)\right)\right]^{4}, \\
& \left(h_{ \pm}^{e}, \widehat{h}_{ \pm}^{e}\right) \in\left[C^{0}\left([0, T] ; H^{1}\left(\Gamma_{ \pm}\right)\right) \cap H^{1}\left(0, T ; L^{2}\left(\Gamma_{ \pm}\right)\right)\right]^{2} .
\end{aligned}
$$

The following compatibility conditions are also assumed:

$$
\left\{\begin{array}{l}
\left.h_{0}^{e}\right|_{t=0}=y_{0}^{e} \text { on } \Gamma_{0},\left.\quad h_{ \pm}^{e}\right|_{t=0}=e^{-1} \frac{\partial y_{0}^{e}}{\partial \nu} \text { on } \Gamma_{ \pm}, \\
\left.\widehat{h}_{0}^{e}\right|_{t=0}=\widehat{y}_{0}^{e}=0 \text { on } \Gamma_{0},\left.\quad \widehat{h}_{ \pm}^{e}\right|_{t=0}=e^{-1} \frac{\partial \widehat{y}_{0}^{e}}{\partial \nu}=0 \text { on } \Gamma_{ \pm} .
\end{array}\right.
$$

Then, there exists a unique solution $y^{e}$ to (2.2) such that

$$
\left(y^{e},\left(y^{e}\right)^{\prime},\left(y^{e}\right)^{\prime \prime}\right) \in C^{0}\left([0, T] ; H^{1}(\Omega) \times H \times\left(V^{e}\right)^{\prime}\right),
$$

(8.4) $\frac{\partial y^{e}}{\partial \nu} \in L^{2}\left(\Sigma_{0}\right),\left(\left.y^{e}\right|_{\Sigma_{ \pm}},\left.\left(y^{e}\right)^{\prime}\right|_{\Sigma_{ \pm}}\right) \in C^{0}\left([0, T] ; H^{1}\left(\Gamma_{ \pm}\right) \times L^{2}\left(\Gamma_{ \pm}\right)\right)$.

Further we have the estimates

$$
\begin{gathered}
\left\|\nabla_{e} y^{e}\right\|_{L^{\infty}\left(0, T ; H^{1}(\Omega)\right)^{3}}+\left\|\left(\partial y^{e} / \partial t\right)\right\|_{L^{\infty}(0, T ; H)} \leq \\
\leq C R_{4}\left(y_{0}^{e}+\tau_{0} \widehat{y}_{0}^{e}, y_{1}^{e}+\tau_{1} \widehat{y}_{1}^{e}\right. \\
\left.h_{0}^{e}+\lambda_{0} \widehat{h}_{0}^{e}, h_{+}^{e}+\lambda_{+} \widehat{h}_{+}^{e}, h_{-}^{e}+\lambda_{-} \widehat{h}_{-}^{e}\right),
\end{gathered}
$$




$$
\begin{aligned}
& \left\|_{L^{2}\left(\Sigma_{0}\right)}+\right\| y^{e}\left\|_{L^{\infty}\left(0, T ; H^{1}\left(\Gamma_{ \pm}\right)\right)}+\right\|\left(y^{e}\right)^{\prime} \|_{L^{\infty}\left(0, T ; L^{2}\left(\Gamma_{ \pm}\right)\right)} \leq \\
& \leq C\left\{R _ { 4 } \left(y_{0}^{e}+\tau_{0} \widehat{y}_{0}^{e}, y_{1}^{e}+\tau_{1} \widehat{y}_{1}^{e},\right.\right. \\
& \left.h_{0}^{e}+\lambda \widehat{h}_{0}^{e}, h_{+}^{e}+\lambda_{+} \widehat{h}_{+}^{e}, h_{-}^{e}+\lambda_{-} \widehat{h}_{-}^{e}\right)+ \\
& +R_{6}\left(\frac{\partial y_{0}^{e}}{\partial z_{3}}+\tau_{0} \frac{\partial \widehat{y}_{0}^{e}}{\partial z_{3}}, \frac{\partial y_{1}^{e}}{\partial z_{3}}+\tau_{1} \frac{\partial \widehat{y}_{1}^{e}}{\partial z_{3}}\right. \\
& \left.\left.\frac{\partial y_{0}^{e}}{\partial z_{3}}+\lambda_{0} \frac{\partial \widehat{h}_{0}^{e}}{\partial z_{3}}, h_{+}^{e}+\lambda_{+} \widehat{h}_{+}^{e}, h_{-}^{e}+\lambda_{-} \widehat{h}_{-}^{e}\right)\right\},
\end{aligned}
$$

where $R_{4}$ and $R_{6}$ were defined by (4.28) and (5.14) respectively.

Remark 8.2. Obviously, we can modify hypothesis (8.1) in the light of Remark 7.4

The purpose of this section is to give a reformulation of the insensitivity conditions (2.7) as an exact controllability problem and thereby construct the sentinel in the form prescribed by (2.6). To this end, let us start by introducing the adjoint state.

\subsection{Adjoint state}

It is classical in Control Theory to introduce the adjoint state in the following manner:

$$
\left\{\begin{array}{l}
\square_{e} q^{e}=0 \quad \text { in } Q \\
q^{e}=\xi_{0}^{e}+w_{0}^{e} \text { on } \Sigma_{0} \quad \text { and } \quad e^{-1} \frac{\partial q^{e}}{\partial \nu}=\xi_{ \pm}^{e}+w_{ \pm}^{e} \quad \text { on } \Sigma_{ \pm} \\
q^{e}(T)=\left(q^{e}\right)^{\prime}(T)=0 \quad \text { in } \Omega
\end{array}\right.
$$

We assume that

$$
\xi_{0}^{e} \in L^{2}\left(\Sigma_{0}\right), \quad \xi_{ \pm}^{e} \in\left(W_{ \pm}^{e}\right)^{\prime} .
$$

We seek therefore $w_{0}^{e}$ and $w_{ \pm}^{e}$ such that insensitivity conditions (2.7) are satisfied and

$$
w_{0}^{e} \in L^{2}\left(\Sigma_{0}\right), \quad w_{ \pm}^{e} \in\left(W_{ \pm}^{e}\right)^{\prime} .
$$

By Theorems 3.3 and 3.4, we know that there exists a unique weak solution $q^{e}$ such that

$$
q^{e} \in C^{0}([0, T] ; H), \quad\left(q^{e}\right)^{\prime} \in C^{0}\left([0, T] ;\left(V^{e}\right)^{\prime}\right) .
$$


Further, it has the property that

$$
\begin{aligned}
& \left\|q^{e}\right\|_{L^{\infty}(0, T ; H)}+\left\|\left(q^{e}\right)^{\prime}\right\|_{L^{\infty}\left(0, T ;\left(V^{e}\right)^{\prime}\right)} \leq \\
& \leq C R_{1}\left(0,0,0, \xi_{0}^{e}+w_{0}^{e}, \xi_{+}^{e}+w_{+}^{e}, \xi_{-}^{e}+w_{-}^{e}\right),
\end{aligned}
$$

where $R_{1}$ is defined by (3.19).

\subsection{First reformulation of the insensitivity conditions}

With the help of the adjoint state $q^{e}$, we rewrite the insensitivity conditions in a different form. To this end, let us differentiate (2.2) with respect to $\tau_{0}$ and $\tau_{1}$. We set $y_{\tau_{j}}^{e}=\left.\left(\partial y^{e} / \partial \tau_{j}\right)\right|_{\tau=0, \lambda=0}$ for $j=0,1$. Then for instance, $y_{\tau_{0}}^{e}$ satisfies

$$
\left\{\begin{array}{l}
\square_{e} y_{\tau_{0}}^{e}=0 \text { in } Q, \\
y_{\tau_{0}}^{e}=0 \text { on } \Sigma_{0} \text { and } e^{-1}\left(\partial y_{\tau_{0}}^{e} / \partial \nu\right)=0 \text { on } \Sigma_{ \pm} \\
y_{\tau_{0}}^{e}(0)=\widehat{y}_{0}^{e} \text { and }\left(y_{\tau_{0}}^{e}\right)^{\prime}(0)=0 \quad \text { in } \Omega .
\end{array}\right.
$$

Multiplying (8.7) by $y_{\tau_{0}}^{e}$, we see that the insensitivity condition $\left.\left(\partial S^{e} / \partial \tau_{0}\right)\right|_{\tau=0, \lambda=0}=0$ is equivalent to

$$
\begin{aligned}
& \left(V^{e}\right)^{\prime}<\left(q^{e}\right)^{\prime}(0), \widehat{y}_{0}^{e}>_{V^{e}}=0, \\
& \forall \widehat{y}_{0}^{e} \in V^{e} \text { satisfying } \frac{\partial \widehat{y}_{0}^{e}}{\partial \nu}=0 \text { on } \Gamma_{ \pm} .
\end{aligned}
$$

Since such functions are dense in $V^{e}$, above condition becomes

$$
\left(q^{e}\right)^{\prime}(0)=0 .
$$

Likewise, multiplying (8.7) by $y_{\tau_{1}}^{e}$, we can see that the second insensitivity condition in (2.7) can be rewritten as

$$
q^{e}(0)=0
$$

\subsection{Transformation to an exact controllability problem}

In this paragraph, we reformulate the insensitivity conditions (8.14), (8.15) as a problem of exact controllability. To this end, let us decompose the solution $q^{e}$ of (8.7) as follows: $q^{e}=u^{e}+v^{e}$, where $u^{e}, v^{e}$ are respective weak solutions of

$$
\left\{\begin{array}{l}
\square_{e} u^{e}=0 \quad \text { in } Q \\
u^{e}=\xi_{0}^{e} \text { on } \Sigma_{0} \text { and } e^{-1}\left(\partial u^{e} / \partial \nu\right)=\xi_{ \pm}^{e} \quad \text { on } \Sigma_{ \pm} \\
u^{e}(T)=\left(u^{e}\right)^{\prime}(T)=0 \quad \text { in } \Omega .
\end{array}\right.
$$




$$
\left\{\begin{array}{l}
\square_{e} v^{e}=0 \text { in } Q \\
v^{e}=w_{0}^{e} \text { on } \Sigma_{0} \text { and } e^{-1}\left(\partial v^{e} / \partial \nu\right)=w_{ \pm}^{e} \text { on } \Sigma_{ \pm} \\
v^{e}(T)=\left(v^{e}\right)^{\prime}(T)=0 \text { in } \Omega
\end{array}\right.
$$

The insensitivity conditions (8.14) and (8.15) are then clearly rewritten as:

$$
v^{e}(0)=-u^{e}(0) \text { in } \Omega, \quad\left(v^{e}\right)^{\prime}(0)=-\left(u^{e}\right)^{\prime}(0) \text { in } \Omega .
$$

Finding $w_{0}^{e}, w_{ \pm}^{e}$ such that (8.17), (8.18) are satisfied is obviously an exact controllability problem. This has been studied by Saint Jean Paulin \& Vanninathan [8]. We will recall in $\S 8.4$ some relevant results from this work, along with some improvements.

\subsection{Study of the exact controllability problem}

Following Hilbert Uniqueness Method (HUM) (cf. Lions [6]), the solvability of problem (8.17) and (8.18) is transformed into the resolution of an equation associated with a certain operator $\Lambda^{e}$. To define it, let us consider the homogeneous mixed problem

$$
\left\{\begin{array}{l}
\square_{e} \varphi^{e}=0 \text { in } Q, \\
\varphi^{e}=0 \text { on } \Sigma_{0} \text { and } e^{-1}\left(\partial \varphi^{e} / \partial \nu\right)=0 \quad \text { on } \Sigma_{ \pm} \\
\varphi^{e}(0)=\varphi_{0}^{e} \text { and }\left(\varphi^{e}\right)^{\prime}(0)=\varphi_{1}^{e} \quad \text { in } \Omega
\end{array}\right.
$$

where the initial condition $\left(\varphi_{0}^{e}, \varphi_{1}^{e}\right)$ is taken in the space $V^{e} \times H$. Hence, we know by Theorem 3.1 that there exists a unique solution $\varphi^{e}$ such that

$$
\begin{aligned}
& \left(\varphi^{e},\left(\varphi^{e}\right)^{\prime},\left(\varphi^{e}\right)^{\prime \prime}\right) \in C^{0}\left([0, T] ; V^{e} \times H \times\left(V^{e}\right)^{\prime}\right) \\
& \int_{\Sigma_{0}}\left(\frac{\partial \varphi^{e}}{\partial \nu}\right)^{2} d \sigma d t+\left|\int_{\Sigma_{+}}\left\{\left(\frac{\partial \varphi^{e}}{\partial t}\right)^{2}-\frac{\partial \varphi^{e}}{\partial z_{\alpha}} \frac{\partial \varphi^{e}}{\partial z_{\alpha}}\right\} d \sigma d t\right|+ \\
& +\left|\int_{\Sigma_{-}}\left\{\left(\frac{\partial \varphi^{e}}{\partial t}\right)^{2}-\frac{\partial \varphi^{e}}{\partial z_{\alpha}} \frac{\partial \varphi^{e}}{\partial z_{\alpha}}\right\} d \sigma d t\right| \leq C T E^{e}\left(\varphi^{e} ; 0\right) .
\end{aligned}
$$

Above estimate is generally known as Direct Inequality. 
Let us now consider finite energy solution $\theta^{e}$ of

$$
\left\{\begin{array}{l}
\square_{e} \theta^{e}=F^{e} \quad \text { in } Q, \\
\theta^{e}=0 \text { on } \Sigma_{0} \text { and } e^{-1}\left(\partial \theta^{e} / \partial \nu\right)=0 \quad \text { on } \Sigma_{ \pm}, \\
\theta^{e}(0)=\theta_{0}^{e} \text { and }\left(\theta^{e}\right)^{\prime}(0)=\theta_{1}^{e} \quad \text { in } \Omega .
\end{array}\right.
$$

Using the multipliers (3.13) and (5.9), we deduce from (7.5) the following inequality, which is a generalization of (8.21).

$$
\begin{aligned}
& \left|\int_{\Sigma_{0}}\left(\partial \varphi^{e} / \partial \nu\right)\left(\partial \theta^{e} / \partial \nu\right) d \sigma d t\right|+ \\
& +\left|\int_{\Sigma_{+}}\left\{\left(\varphi^{e}\right)^{\prime}\left(\theta^{e}\right)^{\prime}-\left(\partial \varphi^{e} / \partial z_{\alpha}\right)\left(\partial \theta^{e} / \partial z_{\alpha}\right)\right\} d \sigma d t\right|+ \\
& +\left|\int_{\Sigma_{-}}\left\{\left(\varphi^{e}\right)^{\prime}\left(\theta^{e}\right)^{\prime}-\left(\partial \varphi^{e} / \partial z_{\alpha}\right)\left(\partial \theta^{e} / \partial z_{\alpha}\right)\right\} d \sigma d t\right| \leq \\
& \leq C T E^{e}\left(\varphi^{e} ; 0\right)^{1 / 2}\left\{E^{e}\left(\theta^{e} ; 0\right)^{1 / 2}+\left\|F^{e}\right\|_{L^{1}(0, T ; H)}\right\} .
\end{aligned}
$$

Above inequality in particular shows that the bilinear form

$$
\int_{\Sigma_{+} \cup \Sigma_{-}}\left\{\left(\varphi^{e}\right)^{\prime}\left(\theta^{e}\right)^{\prime}-\left(\frac{\partial \varphi^{e}}{\partial z_{\alpha}}\right)\left(\frac{\partial \theta^{e}}{\partial z_{\alpha}}\right)\right\} d \sigma d t
$$

is well defined for all finite energy solutions. Indeed, the definition of the bilinear form can be obtained from the right-hand side of (7.5). We interpret (8.24) as the duality action

$$
-\int_{\Sigma_{+} \cup \Sigma_{-}}\left(\frac{\partial^{2} \varphi^{e}}{\partial t^{2}}-\frac{\partial^{2} \varphi^{e}}{\partial z_{\alpha} \partial z_{\alpha}}\right) \theta^{e} d \sigma d t
$$

Recalling that $W_{ \pm}^{e}$ denote the spaces of the traces on $\Sigma_{ \pm}$of solutions with finite energy, we see that the above procedure shows that $\left(\left(\partial^{2} \varphi^{e} / \partial t^{2}\right)-\right.$ $\left.\left(\partial^{2} \varphi^{e} / \partial z_{\alpha} \partial z_{\alpha}\right)\right)\left.\right|_{\Sigma_{ \pm}} \in\left(W_{ \pm}^{e}\right)^{\prime}$. Further, (8.23) shows that

$$
\left\|\frac{\partial^{2} \varphi^{e}}{\partial t^{2}}-\frac{\partial^{2} \varphi^{e}}{\partial z_{\alpha} \partial z_{\alpha}}\right\|_{\left(W_{ \pm}^{e}\right)^{\prime}} \leq C T E^{e}\left(\varphi^{e} ; 0\right)^{1 / 2} .
$$

Let us now pass on to what is referred to as Inverse Inequality and it is as follows. 
Proposition 8.3. There exists $T_{\star}>0$ and a constant $C>0$, both independent of e, such that $\forall T \geq T^{\star}$, we have

$$
\begin{aligned}
T E^{e}\left(\varphi^{e} ; 0\right) & \leq C\left[\int_{\Sigma_{0}}\left(\frac{\partial \varphi^{e}}{\partial \nu}\right)^{2} d \sigma d t+\right. \\
& \left.+\int_{\Sigma_{+} \cup \Sigma_{-}}\left\{\left(\frac{\partial \varphi^{e}}{\partial t}\right)^{2}-\frac{\partial \varphi^{e}}{\partial z_{\alpha}} \frac{\partial \varphi^{e}}{\partial z_{\alpha}}\right\} d \sigma d t\right] .
\end{aligned}
$$

Before giving the proof, let us observe that there is no modulus in the second integral on the right-hand side of the inequality.

Proof. We multiply (8.19) by $m_{k}\left(\partial \varphi^{e} / \partial z_{k}\right)$ with $m_{k}=z_{k}-z_{k}^{0}$ where $z^{0}=\left(z_{1}^{0}, z_{2}^{0}, 0\right)$ is a point a priori fixed. After some standard computations, we arrive at (see relation (5.6) of Saint Jean Paulin \& Vanninathan $[8])$ :

$$
\begin{aligned}
& \frac{1}{2} \int_{Q}\left\{\left(\frac{\partial \varphi^{e}}{\partial t}\right)^{2}+\left|\nabla_{e} \varphi^{e}\right|^{2}\right\} d z d t= \\
& =\frac{1}{2} \int_{\Sigma_{0}}\left(m_{\alpha} \nu_{\alpha}\right)\left(\frac{\partial \varphi^{e}}{\partial \nu}\right)^{2} d \sigma d t+ \\
& +\frac{1}{4} \int_{\Sigma_{+} \cup \Sigma_{-}}\left\{\left(\frac{\partial \varphi^{e}}{\partial t}\right)^{2}-\frac{\partial \varphi^{e}}{\partial z_{\alpha}} \frac{\partial \varphi^{e}}{\partial z_{\alpha}}\right\} d \sigma d t- \\
& -\left[\int_{\Omega} \frac{\partial \varphi^{e}}{\partial t}\left(\varphi^{e}+m_{k} \frac{\partial \varphi^{e}}{\partial z_{k}}\right) d z\right]_{0}^{T} .
\end{aligned}
$$

Now, we estimate the last term in a fashion different from Saint Jean Paulin \& Vanninathan [8] and this yields a marked improvement. Indeed, we have

$$
\begin{aligned}
& \left|\int_{\Omega} \frac{\partial \varphi^{e}}{\partial t}\left(\varphi^{e}+m_{k} \frac{\partial \varphi^{e}}{\partial z_{k}}\right) d z\right| \leq \frac{1}{2} \int_{\Omega}\left(\frac{\partial \varphi^{e}}{\partial t}\right)^{2} d z+ \\
& +\frac{1}{2} \int_{\Omega}\left(\varphi^{e}+m_{k} \frac{\partial \varphi^{e}}{\partial z_{k}}\right)^{2} d z \\
& \leq C E^{e}\left(\varphi^{e} ; 0\right), \text { by Poincaré Inequality. }
\end{aligned}
$$

If we apply this inequality in (8.28), then classical arguments imply (8.27). 
The next step in H.U.M is to consider the backward problem written formally as follows:

$$
\left\{\begin{array}{l}
\square_{e} \psi^{e}=0 \quad \text { in } Q \\
\psi^{e}=\frac{\partial \varphi^{e}}{\partial \nu} \quad \text { on } \Sigma_{0}, e^{-1} \frac{\partial \psi^{e}}{\partial \nu}=e\left(\frac{\partial^{2} \varphi^{e}}{\partial t^{2}}-\frac{\partial^{2} \varphi^{e}}{\partial z_{\alpha} \partial z_{\alpha}}\right) \quad \text { on } \Sigma_{ \pm} \\
\psi^{e}(T)=\left(\psi^{e}\right)^{\prime}(T)=0 \quad \text { in } \Omega
\end{array}\right.
$$

where $\varphi^{e}$ is the solution of (8.19) with initial conditions $\left(\varphi_{0}^{e}, \varphi_{1}^{e}\right) \in$ $V^{e} \times H$.

To give a rigorous formulation of (8.30), let us multiply (8.22) by $\psi^{e}$. Using (7.5) and multipliers $m_{k}^{0}$ are such that

$m_{\alpha}^{0}$ independent of $z_{3}, \quad m_{\alpha}^{0}=0$ on $\Gamma_{0}, \quad m_{3}^{0}=\nu_{3}$ on $\Gamma_{ \pm}$, we obtain

$$
\begin{aligned}
& \int_{Q} F^{e} \psi^{e} d z d t-\int_{\Omega}\left\{\frac{\partial \psi^{e}}{\partial t}(0) \theta_{0}^{e}-\psi^{e}(0) \theta_{1}^{e}\right\} d z= \\
& -\int_{\Sigma_{0}} \frac{\partial \varphi^{e}}{\partial \nu} \frac{\partial \theta^{e}}{\partial \nu} d \sigma d t+\int_{Q} F^{e} m_{k}^{0} \frac{\partial \varphi^{e}}{\partial z_{k}} d z d t- \\
& -\left[\int_{\Omega}\left\{\frac{\partial \theta^{e}}{\partial t} m_{k}^{0} \frac{\partial \varphi^{e}}{\partial z_{k}}+\frac{\partial \varphi^{e}}{\partial t} m_{k}^{0} \frac{\partial \theta^{e}}{\partial z_{k}}\right\} d z\right]_{0}^{T}- \\
& -\int_{Q} \frac{\partial m_{k}^{0}}{\partial z_{k}}\left\{\frac{\partial \varphi^{e}}{\partial t} \frac{\partial \theta^{e}}{\partial t}-\nabla_{e} \varphi^{e} \cdot \nabla_{e} \theta^{e}\right\} d z d t- \\
& -\int_{Q} \frac{\partial m_{k}^{0}}{\partial z_{\alpha}}\left\{\frac{\partial \theta^{e}}{\partial z_{\alpha}} \frac{\partial \varphi^{e}}{\partial z_{k}}+\frac{\partial \varphi^{e}}{\partial z_{\alpha}} \frac{\partial \theta^{e}}{\partial z_{k}}\right\} d z d t- \\
& -2 e^{-2} \int_{Q} \frac{\partial m_{3}^{0}}{\partial z_{3}} \frac{\partial \theta^{e}}{\partial z_{3}} \frac{\partial \varphi^{e}}{\partial z_{3}} d z d t .
\end{aligned}
$$

We demand that relation (8.31) is satisfied for all $F^{e} \in L^{1}(0, T ; H)$ and for all corresponding solutions $\theta^{e}$ of (8.22). This is the rigorous formulation of the problem (8.30). It follows from (8.31) (cf. Saint Jean Paulin \& Vanninathan [8]) that there is a unique solution to (8.30) such that

(8.32) $\left(\psi^{e},\left(\psi^{e}\right)^{\prime}\right) \in L^{\infty}\left(0, T ; H \times\left(V^{e}\right)^{\prime}\right),\left(\left(\psi^{e}\right)^{\prime}(0), \psi^{e}(0)\right) \in\left(V^{e}\right)^{\prime} \times H$.

Further, we have the estimate

$$
\begin{aligned}
& \left\|\left(\psi^{e},\left(\psi^{e}\right)^{\prime}\right)\right\|_{L^{\infty}\left(0, T ; H \times\left(V^{e}\right)^{\prime}\right)}+ \\
& +\left\|\left(\left(\psi^{e}\right)^{\prime}(0), \psi^{e}(0)\right)\right\|_{\left(V^{e}\right)^{\prime} \times H} \leq C E^{e}\left(\varphi^{e} ; 0\right)^{1 / 2} .
\end{aligned}
$$


With the help of the backward problem, we are now in a position to introduce the operator $\Lambda^{e}$ which will solve the exact controllability problem. Let us define

$$
\begin{aligned}
& \Lambda^{e}: V^{e} \times H \longrightarrow\left(V^{e}\right) \times H \\
& \left(\varphi_{0}^{e}, \varphi_{1}^{e}\right) \longmapsto\left(\left(\psi^{e}\right)^{\prime}(0),-\psi^{e}(0)\right) .
\end{aligned}
$$

It is verified that, if $T \geq T^{\star}$, then $\Lambda^{e}$ is an isomorphism and in fact we have

$$
\left(V^{e}\right)^{\prime} \times H<\Lambda^{e}\left(\varphi_{0}^{e}, \varphi_{1}^{e}\right),\left(\varphi_{0}^{e}, \varphi_{1}^{e}\right)>_{V^{e} \times H} \geq C\left\|\left(\varphi_{0}^{e}, \varphi_{1}^{e}\right)\right\|_{V^{e} \times H}^{2}
$$

This is a consequence of the Inverse Inequality stated in Proposition 8.3.

Thanks to the isomorphism $\Lambda^{e}$, we can now go back to the exact controllability problem (8.17), (8.18) and solve it. In fact, it is enough to observe that $\left(\left(u^{e}\right)^{\prime}(0), u^{e}(0)\right) \in\left(V^{e}\right)^{\prime} \times H$ and the problem (8.17) coincides with (8.30) where the boundary controls are given by

$$
\begin{gathered}
w_{0}^{e}=\left(\partial \varphi^{e} / \partial \nu\right) \quad \text { on } \Sigma_{0} \\
w_{ \pm}^{e}=e\left(\left(\varphi^{e}\right)^{\prime \prime}-\left(\partial^{2} \varphi^{e} / \partial z_{\alpha} \partial z_{\alpha}\right)\right) \quad \text { on } \Sigma_{ \pm} .
\end{gathered}
$$

Here $\varphi^{e}$ is the solution of (8.19) with initial conditions defined by

$$
\Lambda^{e}\left(\varphi_{0}^{e}, \varphi_{1}^{e}\right)=\left(-\left(u^{e}\right)^{\prime}(0), u^{e}(0)\right) .
$$

Let us terminate this paragraph by writing down the estimates that easily follow from our analysis. First, it is an easy consequence of (8.35) that

$$
\left\|\left(\varphi_{0}^{e}, \varphi_{1}^{e}\right)\right\|_{V^{e} \times H} \leq C\left\|\left(-\left(u^{e}\right)^{\prime}(0), u^{e}(0)\right)\right\|_{\left(V^{e}\right)^{\prime} \times H} .
$$

Next the inequalities (8.21) and (8.26) show that $\left(w_{0}^{e}, w_{ \pm}^{e}\right) \in L^{2}\left(\Sigma_{0}\right) \times$ $\left(W_{ \pm}^{e}\right)^{\prime}$ and we have the estimate

$$
\begin{aligned}
& \left\|w_{0}^{e}\right\|_{L^{2}\left(\Sigma_{0}\right)}+e^{-1}\left\|w_{+}^{e}\right\|_{\left(W_{+}^{e}\right)^{\prime}}+e^{-1}\left\|w_{-}^{e}\right\|_{\left(W_{-}^{e}\right)^{\prime}} \leq \\
& \leq C T\left\|\left(-\left(u^{e}\right)^{\prime}(0), u^{e}(0)\right)\right\|_{\left(V^{e}\right)^{\prime} \times H} .
\end{aligned}
$$




\subsection{Definition of the boundary sentinel}

Let us recall that, for given functions $\left(\xi_{0}^{e}, \xi_{ \pm}^{e}\right) \in L^{2}\left(\Sigma_{0}\right) \times\left(W_{ \pm}^{e}\right)^{\prime}$, the boundary sentinel is defined by (cf. (2.6)):

$$
\begin{aligned}
& S^{e}(\lambda, \tau)=e \int_{\Sigma_{0}}\left(\xi_{0}^{e}+w_{0}^{e}\right)\left(\partial y^{e} / \partial \nu\right)(\lambda, \tau) d \sigma d t- \\
& -\int_{\Sigma_{+}}\left(\xi_{+}^{e}+w_{+}^{e}\right) y^{e}(\lambda, \tau) d \sigma d t-\int_{\Sigma_{-}}\left(\xi_{-}^{e}+w_{-}^{e}\right) y^{e}(\lambda, \tau) d \sigma d t .
\end{aligned}
$$

We are now in a position to answer the question of finding $w_{0}^{e}, w_{ \pm}^{e}$ such that the sentinel defined by (2.6) satisfies the insensitivity conditions (2.7). Indeed, $w_{0}^{e}, w_{ \pm}^{e}$ are given by (8.36), (8.37) respectively, and so the boundary sentinel takes the form

$$
\begin{aligned}
& S^{e}(\lambda, \tau)=e \int_{\Sigma_{0}} \xi_{0}^{e} \frac{\partial y^{e}}{\partial \nu}(\lambda, \tau) d \sigma d t-\int_{\Sigma_{+}} \xi_{+}^{e} y^{e}(\lambda, \tau) d \sigma d t- \\
& -\int_{\Sigma_{-}} \xi_{-}^{e} y^{e}(\lambda, \tau) d \sigma d t+e \int_{\Sigma_{0}}\left(\partial \varphi^{e} / \partial \nu\right)\left(\partial y^{e} / \partial \nu\right)(\lambda, \tau) d \sigma d t+ \\
& +e \int_{\Sigma_{+} \cup \Sigma_{-}}\left(\left(\varphi^{e}\right)^{\prime}\left(y^{e}\right)^{\prime}-\left(\partial \varphi^{e} / \partial z_{\alpha}\right)\left(\partial y^{e} / \partial z_{\alpha}\right)\right) d \sigma d t
\end{aligned}
$$

Let us remember that the mapping $\left(\xi_{0}^{e}, \xi_{ \pm}^{e}\right) \mapsto \varphi^{e}$ is defined by (8.16), (8.38). We end this paragraph by observing that the sum of the last two terms can be obtained from the relation (7.5) with the following choice of multipliers:

$$
\begin{aligned}
& m_{1}, m_{2} \text { independent of } z_{3}, \\
& m_{\beta}=\nu_{\beta} \text { on } \Gamma_{0}, \beta=1,2, m_{3}=\nu_{3} \text { on } \Gamma_{ \pm} .
\end{aligned}
$$

In particular, this shows that $e^{-1} w_{ \pm}^{e}$ acts not only on homogeneous solutions $\theta^{e}$, but also on non-homogeneous solutions $y^{e}$ of finite energy.

\section{Stealthiness conditions}

In general, the boundary sentinel $S^{e}$, defined in (8.41), (which satisfies, by our construction, the insensitivity conditions) depends on the pollution terms $\lambda_{0} \widehat{h}_{0}^{e}, \lambda_{ \pm} \widehat{h}_{ \pm}^{e}$ since the solution $y^{e}(\lambda, \tau)$ does so. In order 
to bring out this dependence, at least up to first order in $\lambda_{0}, \lambda_{ \pm}$, let us do a Taylor expansion:

$$
S^{e}(\lambda, \tau)=S^{e}(0,0)+\lambda \cdot\left(\partial S^{e} / \partial \lambda\right)(0,0)+\cdots
$$

From this, it is clear that $S^{e}(\lambda, \tau)$ contains no information on the pollution terms (at least up to first order) if

$$
\frac{\partial S^{e}}{\partial \lambda_{0}}(0,0)=0, \quad \frac{\partial S^{e}}{\partial \lambda_{+}}(0,0)=0, \quad \frac{\partial S^{e}}{\partial \lambda_{-}}(0,0)=0 .
$$

If the pollution terms are such that (9.2) holds for all sentinels $S^{e}$ of the form (2.6) (or equivalently for all choices $\xi_{0}^{e}, \xi_{ \pm}^{e}$ ), then such pollution terms are called stealthy.

Our objective, in this section, is to write down explicitly the condition (9.2) for the system (2.2) under investigation and thereby characterize all stealthy pollutions. To achieve this, let us differentiate (2.2) with respect to $\lambda_{0}, \lambda_{ \pm}$. We set

$$
y_{\lambda_{0}}^{e}=\left(\partial y^{e} / \partial \lambda_{0}\right)(0,0), \quad y_{\lambda_{ \pm}}^{e}=\left(\partial y^{e} / \partial \lambda_{ \pm}\right)(0,0) .
$$

For instance $y_{\lambda_{0}}^{e}$ satisfies the system

$$
\left\{\begin{array}{l}
\square_{e} y_{\lambda_{0}}^{e}=0 \text { in } Q, \\
y_{\lambda_{0}}^{e}=\widehat{h}_{0}^{e} \text { on } \Sigma_{0} \text { and } e^{-1}\left(\partial y_{\lambda_{0}}^{e} / \partial \nu\right)=0 \text { on } \Sigma_{ \pm}, \\
\left(y_{\lambda_{0}}^{e}\right)(0)=\left(y_{\lambda_{0}}^{e}\right)^{\prime}(0)=0 \text { in } \Omega .
\end{array}\right.
$$

Recall that Theorem 8.1 guarantees the existence and the uniqueness of solutions $y_{\lambda_{0}}^{e}$.

Now the first condition in (9.3) is written as

$$
\begin{aligned}
& e \int_{\Sigma_{0}}\left(\xi_{0}^{e}+w_{0}^{e}\right)\left(\partial y_{\lambda_{0}}^{e} / \partial \nu\right) d \sigma d t- \\
& -\int_{\Sigma_{+}}\left(\xi_{+}^{e}+w_{+}^{e}\right) y_{\lambda_{0}}^{e} d \sigma d t-\int_{\Sigma_{-}}\left(\xi_{-}^{e}+w_{-}^{e}\right) y_{\lambda_{0}}^{e} d \sigma d t=0 .
\end{aligned}
$$

Let us recall that $w_{0}^{e}, w_{ \pm}^{e}$ are defined by (8.36), (8.37) and that they are determined by $\left(\xi_{0}^{e}, \xi_{ \pm}^{e}\right)$ (cf. (8.16), (8.38), (8.19)). In order to make this dependence transparent, we introduce the following operators:

$$
\left\{\begin{aligned}
M^{e}: & L^{2}\left(\Sigma_{0}\right) \times\left(W_{+}^{e}\right)^{\prime} \times\left(W_{-}^{e}\right)^{\prime} \rightarrow\left(V^{e}\right)^{\prime} \times H \\
& M^{e}\left(\xi_{0}^{e}, \xi_{+}^{e}, \xi_{-}^{e}\right)=\left(\left(u^{e}\right)^{\prime}(0),-u^{e}(0)\right) .
\end{aligned}\right.
$$




$$
\left\{\begin{array}{l}
N^{e}: V^{e} \times H \rightarrow L^{2}\left(\Sigma_{0}\right) \times\left(W_{+}^{e}\right)^{\prime} \times\left(W_{-}^{e}\right)^{\prime} \\
N^{e}\left(\varphi_{0}^{e}, \varphi_{1}^{e}\right)= \\
\left(\left.\frac{\partial \varphi^{e}}{\partial \nu}\right|_{\Sigma_{0}},\left.e\left(\frac{\partial^{2} \varphi^{e}}{\partial t^{2}}-\frac{\partial^{2} \varphi^{e}}{\partial z_{\alpha} \partial z_{\alpha}}\right)\right|_{\Sigma_{+}},\left.e\left(\frac{\partial^{2} \varphi^{e}}{\partial t^{2}}-\frac{\partial^{2} \varphi^{e}}{\partial z_{\alpha} \partial z_{\alpha}}\right)\right|_{\Sigma_{-}}\right)
\end{array}\right.
$$

Easy computations show that the adjoint of $M^{e}$ is given by

$$
\left\{\begin{array}{l}
\left(M^{e}\right)^{\star}: V^{e} \times H \rightarrow L^{2}\left(\Sigma_{0}\right) \times W_{+}^{e} \times W_{-}^{e} \\
\left(M^{e}\right)^{\star} \frac{\varphi_{0}^{e}}{\varphi_{1}^{e}}=\left(\left.\frac{\partial \varphi^{e}}{\partial \nu}\right|_{\Sigma_{0}},-\left.e^{-1} \varphi^{e}\right|_{\Sigma_{+}},-\left.e^{-1} \varphi^{e}\right|_{\Sigma_{-}}\right) .
\end{array}\right.
$$

With these definitions, it is quite clear that

$$
\left(w_{0}^{e}, w_{+}^{e}, w_{-}^{e}\right)=-N^{e}\left(\Lambda^{e}\right)^{-1} M^{e}\left(\xi_{0}^{e}, \xi_{+}^{e}, \xi_{-}^{e}\right) .
$$

We can now recast the relation (9.5) as

$$
\begin{aligned}
& <\left(\left.e \frac{\partial y_{\lambda_{0}}^{e}}{\partial \nu}\right|_{\Sigma_{0}},\left.y_{\lambda_{0}}^{e}\right|_{\Sigma_{+}},\left.y_{\lambda_{0}}^{e}\right|_{\Sigma_{-}}\right), \\
& \left(I-N^{e}\left(\Lambda^{e}\right)^{-1} M^{e}\right)\left(\xi_{0}^{e}, \xi_{+}^{e}, \xi_{-}^{e}\right)>=0 .
\end{aligned}
$$

Stealthiness requires that this be true for all $\left(\xi_{0}^{e}, \xi_{+}^{e}, \xi_{-}^{e}\right)$ and thus we obtain

$$
\begin{aligned}
& \left(I-\left(M^{e}\right)^{\star}\left(\Lambda^{e}\right)^{-1}\left(N^{e}\right)^{\star}\right)\left(\left.\left(\partial y_{\lambda_{0}}^{e} / \partial \nu\right)\right|_{\Sigma_{0}},\right. \\
& \left.--\left.e^{-1} y_{\lambda_{0}}^{e}\right|_{\Sigma_{+}},-\left.e^{-1} y_{\lambda_{0}}^{e}\right|_{\Sigma_{-}}\right)=0 .
\end{aligned}
$$

The second and the third relations in (9.3) may be treated in the same manner and we get

$$
\begin{aligned}
& \left(I-\left(M^{e}\right)^{\star}\left(\Lambda^{e}\right)^{-1}\left(N^{e}\right)^{\star}\right)\left(\left.\left(\partial y_{\lambda_{+}}^{e} / \partial \nu\right)\right|_{\Sigma_{0}},\right. \\
& \left.-\left.e^{-1} y_{\lambda_{+}}^{e}\right|_{\Sigma_{+}},-\left.e^{-1} y_{\lambda_{+}}^{e}\right|_{\Sigma_{-}}\right)=0 . \\
& \left(I-\left(M^{e}\right)^{\star}\left(\Lambda^{e}\right)^{-1}\left(N^{e}\right)^{\star}\right)\left(\left.\left(\partial y_{\lambda_{-}}^{e} / \partial \nu\right)\right|_{\Sigma_{0}},\right. \\
& \left.-\left.e^{-1} y_{\lambda_{-}}^{e}\right|_{\Sigma_{+}},-\left.e^{-1} y_{\lambda_{-}}^{e}\right|_{\Sigma_{-}}\right)=0 .
\end{aligned}
$$

Thus (9.11) - (9.13) constitute the necessary and sufficient conditions for the stealthiness of the pollution terms $\lambda_{0} \widehat{h}_{0}^{e}$ and $\lambda_{ \pm} \widehat{h}_{ \pm}^{e}$. If we use the form of the image of $\left(M^{e}\right)^{\star}$ (cf. (9.8)) in the above relations, we arrive at the following conclusion. 
Theorem 9.1. The pollution terms $\lambda_{0} \widehat{h}_{0}^{e}, \lambda_{ \pm} \widehat{h}_{ \pm}^{e}$ are stealthy iff there exist finite energy solutions $\chi_{0}^{e}, \chi_{+}^{e}, \chi_{-}^{e}$ satisfying the overdetermined systems

$$
\begin{gathered}
\left\{\begin{array}{l}
\square_{e} \chi_{0}^{e}=\text { in } Q, \\
\chi_{0}^{e}=\widehat{h}_{0}^{e} \text { and }\left(\partial \widehat{\chi}_{0}^{e} / \partial \nu\right)=0 \text { on } \Sigma_{0}, \\
\chi_{0}^{e}=e^{-1}\left(\partial \chi_{0}^{e} / \partial \nu\right)=0 \text { on } \Sigma_{+} \cup \Sigma_{-} .
\end{array}\right. \\
\left\{\begin{array}{l}
\square_{e} \chi_{ \pm}^{e}=0 \text { in } Q, \\
\chi_{ \pm}^{e}=\left(\frac{\partial \chi_{ \pm}^{e}}{\partial \nu}\right)=0 \text { on } \Sigma_{0}, \\
e^{-1} \frac{\partial \chi_{+}^{e}}{\partial \nu}= \begin{cases}\widehat{h}_{+}^{e} \text { on } \Sigma_{+}, \\
0 \text { on } \Sigma_{-},\end{cases} \\
\chi_{ \pm}^{e}=0 \text { ond } \Sigma_{+} \cup \Sigma_{-} .
\end{array}\right.
\end{gathered}
$$

Acknowledgements. The authors are very grateful to the referee for several helpful comments and remarks.

\section{References}

[1] P. GRISVARD, Behaviour of the solutions of elliptic boundary value problems in polygonal or polyhedral domains, p.207-274, in Numerical Solution of Partial Differential Equations - III, B. Hubbard (Ed.), Academic Press, New York, 1976.

[2] P. GRISVARD, Contrôlabilité exacte des solutions de l'équation des ondes en présence de singularités, J. Math. Pures et Appl., 68, p.215-259, 1989.

[3] I. LASIECKA, J.L. LIONS \& R. TRIGGIANI, Non-homogeneous boundary value problems for second order hyperbolic operators, J. Math. Pures et Appl., 65, p.149-192, 1986.

[4] I. LASIECKA \& R. TRIGGIANI, Recent Advances in Regularity of Second Order Hyperbolic Mixed Problems and Applications, p.104-162, in Dynamics Reported 3 (New Series), C.K.R.T. Jones, U. Kirchgraber, H.O. Walther (Eds.), Springer-Verlag, Berlin, 1994.

[5] J.L. LIONS, Optimal control of Systems Governed by Partial Differential Equations, Springer-Verlag, Berlin, 1971.

[6] J.L. LIONS, Contrôlabilité exacte, perturbations et stabilisation de systèmes distribués, Tomes 1, 2, Collection RMA \# 8, 9, Masson, Paris, 1988. 
[7] J.L. LIONS, Sentinelles pour les systèmes distribués à données incomplètes, Collection RMA \# 21, Masson, Paris, 1992.

[8] J. SAINT JEAN PAULIN \& M. VANNINATHAN, Exact Controllability of Vibrations of Thin Bodies, Portugaliae Mathematica, 51 (3), p.421-453, 1994.

J. Saint Jean Paulin

Département de Mathématiques

Université de Metz, Ile du Saulcy

57045 Metz Cedex 01

France

M. Vanninathan

TIFR centre, P.O. Box 1234

Bangalore 560012

Recibido: 8 de Septiembre de 1999

India

Revisado: 27 de Septiembre de 2000 